The Astrophysical JouRnAL, 547:1077-1089, 2001 February 1

(C) 2001. The American Astronomical Society. All rights reserved. Printed in U.S.A.

\title{
SPECTRAL ENERGY DISTRIBUTIONS OF PASSIVE T TAURI AND HERBIG Ae DISKS: GRAIN MINERALOGY, PARAMETER DEPENDENCES, AND COMPARISON WITH INFRARED SPACE OBSERVATORY LWS OBSERVATIONS
}

\author{
E. I. Chiang, ${ }^{1,2,3}$ M. K. Joung, ${ }^{3,4}$ M. J. Creech-EaKman, ${ }^{5,6}$ C. Qi, ${ }^{6}$ J. E. Kessler, ${ }^{7}$ \\ G. A. BlaKe, ${ }^{6,7}$ AND E. F. VAN DishoeCK ${ }^{8}$ \\ Received 2000 August 1; accepted 2000 September 21
}

\begin{abstract}
We improve upon the radiative, hydrostatic equilibrium models of passive circumstellar disks constructed by Chiang \& Goldreich. New features include (1) an account for a range of particle sizes, (2) employment of laboratory-based optical constants of representative grain materials, and (3) numerical solution of the equations of radiative and hydrostatic equilibrium within the original two-layer (disk surface plus disk interior) approximation. We systematically explore how the spectral energy distribution (SED) of a face-on disk depends on grain size distributions, disk geometries and surface densities, and stellar photospheric temperatures. Observed SEDs of three Herbig Ae and two T Tauri stars, including spectra from the Long Wavelength Spectrometer (LWS) aboard the Infrared Space Observatory (ISO), are fitted with our models. Silicate emission bands from optically thin, superheated disk surface layers appear in nearly all systems. Water ice emission bands appear in LWS spectra of two of the coolest stars. Infrared excesses in several sources are consistent with significant vertical settling of photospheric grains. While this work furnishes further evidence that passive reprocessing of starlight by flared disks adequately explains the origin of infrared-to-millimeter wavelength excesses of young stars, we emphasize by explicit calculations how the SED alone does not provide sufficient information to constrain particle sizes and disk masses uniquely.
\end{abstract}

Subject headings: accretion, accretion disks - circumstellar matter - radiative transfer stars: individual (MWC 480, HD 36112, CQ Tauri, LkCa 15, AA Tauri) stars: pre-main-sequence

\section{INTRODUCTION}

The energetics of the outermost regions of isolated disks surrounding $\mathrm{T}$ Tauri and Herbig Ae stars is dominated by passive reprocessing of central starlight. While many protostellar disks are actively accreting (see, e.g., the review by Calvet, Hartmann, \& Strom 2000), effects of viscous dissipation on disk spectra manifest themselves most strongly in the immediate vicinities of the central stars, i.e., in the steepest portions of their gravitational potential wells. Simple scaling laws illustrate the relative importance of external irradiation versus accretion luminosity. The local viscous luminosity per unit disk area decreases as $1 / a^{3}$, where $a$ is the stellocentric distance. By contrast, the flux of central stellar radiation striking the disk drops more slowly as $(\sin \alpha) / a^{2}$, where $\alpha$ is the angle at which starlight grazes the disk surface. Vertical hydrostatic equilibrium normally

\footnotetext{
${ }^{1}$ Hubble Fellow.

${ }^{2}$ Institute for Advanced Study, School of Natural Sciences, Einstein Drive, Princeton, NJ 08540; chiang@ias.edu.

${ }^{3}$ Theoretical Astrophysics, California Institute of Technology 130-33, Pasadena, CA 91125.

${ }^{4}$ Department of Astronomy, Columbia University, New York, NY 10027;moo@astro.columbia.edu.

${ }^{5}$ Jet Propulsion Lab, MS 171-113, Pasadena, CA 91109; mce@ huey.jpl.nasa.gov.

${ }^{6}$ Division of Geological and Planetary Sciences, California Institute of Technology 150-21, Pasadena, CA 91125; qch@gps.caltech.edu, gab@ gps.caltech.edu.

${ }^{7}$ Division of Chemistry and Chemical Engineering, California Institute of Technology, Pasadena, CA 91125; kessler@gps.caltech.edu.

${ }^{8}$ Sterrewacht Leiden, P.O. Box 9513, 2300 RA, Leiden, Netherlands; ewine@strw.leidenuniv.nl.
}

ensures that disks flare outward such that $\alpha$ is a slowly increasing function of $a$ for $a \gg R_{*}$, where $R_{*}$ is the stellar radius (see, e.g., Kenyon \& Hartmann 1987). Hence, there is always a disk radius outside of which the energy from stellar illumination outweighs that of midplane accretion; in the extreme case that the central star derives its luminosity wholly from accretion, this transition radius is roughly 1 AU. The spectral energy distributions (SEDs) of young star/ disk systems longward of $\sim 10 \mu \mathrm{m}$ should thus closely approximate those of passively heated disks, even when accretion is ongoing.

Hydrostatic, radiative equilibrium models of passive $T$ Tauri disks are derived by Chiang \& Goldreich (1997, hereafter CG97). The passive disk divides naturally into two regions: a surface layer that contains dust grains directly exposed to central starlight, and a cooler interior that is encased and diffusively heated by the surface (Calvet et al. 1991; Malbet \& Bertout 1991; CG97; D’Alessio et al. 1998). CG97 compute SEDs of passive disks viewed face-on and employ their model to satisfactorily fit the flattish infrared excess and millimeter wavelength emission of the $T$ Tauri star GM Aur. The optically thin, superheated surface layer is shown to be the natural seat of silicate emission lines (see also Calvet et al. 1992).

In a second paper, Chiang \& Goldreich (1999, hereafter CG99) compute SEDs of passive T Tauri disks viewed at arbitrary inclinations. They point out that the spectrum of a nearly edge-on disk is that of a class I source for which $v F_{v}$ rises from 2 to $10 \mu \mathrm{m}$ (Lada \& Wilking 1984; Lada 1987). In general, class I sources are best described by a combination of an inclined, passively heated disk and a dusty bipolar outflow or partially evacuated envelope. The fraction of 
class I T Tauri spectra that represent limiting cases of simple, isolated, inclined disks is small (D'Alessio et al. 1999) but nonzero (CG99).

This third paper in our series on passive protostellar disks extends our work in three directions:

1. We refine equilibrium, two-layer models of passive disks by (1) accounting for a range of particle sizes, (2) employing laboratory-based optical constants of a suite of circumstellar grain materials, and (3) solving numerically the equations of radiative and hydrostatic equilibrium within our original two-layer approximation.

2. We systematically explore how the SED of a face-on disk depends on grain size distributions, disk geometries and surface densities, and stellar photospheric temperatures. Physical explanations are provided for all observed behaviors of the SED.

3. We employ our refined face-on models to fit observed SEDs of 3 Herbig Ae (HAe) and $2 \mathrm{~T}$ Tauri stars. These observations include new spectra between 43 and $195 \mu \mathrm{m}$ from the Long Wavelength Spectrometer (LWS) aboard the Infrared Space Observatory (ISO) (Creech-Eakman et al. 2000 , in preparation). The uniqueness of our fitted values of disk parameters is assessed, and evidence for emission lines from superheated silicates and ices is reviewed.

The input parameters and basic equations governing our refined standard model are detailed in $\S 2$. Results, including a systematic exploration of how the SED varies in input parameter space, are presented in $\S 3$. Model fits to observations are supplied and critically examined in $\S 4$. There we also compare our results to recent modeling efforts by Miroshnichenko et al. (1999). Finally, we summarize our findings in $\S 5$.

\section{REFINED MODEL}

\subsection{Input Parameters}

Table 1 lists the input parameters of our refined model. Figure 1 exhibits schematically the zones of varying grain composition in both the disk surface and disk interior. For

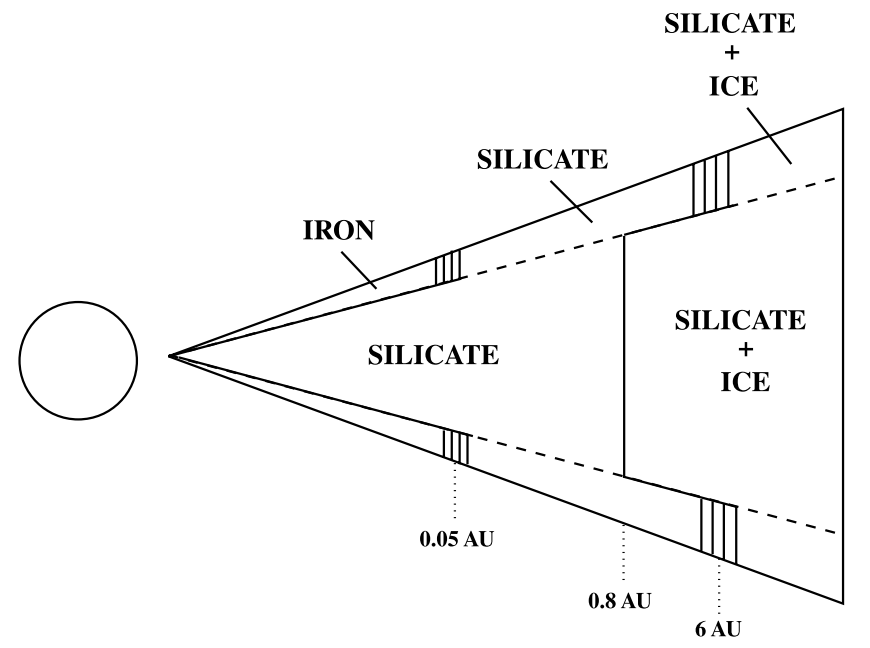

FIG. 1.-Schematic of zones of grain compositions (iron $\rightarrow$ amorphous olivine $\rightarrow$ amorphous olivine mantled with water ice) for both the disk surface and interior. The numerous vertical lines drawn in the surface indicate that there we account for how grains of different sizes sublimate at different stellocentric distances. Dashed lines divide the superheated surface layers from the disk interior. Dotted lines mark locations of condensation boundaries for the refined standard model only.

ease of computation, and for want of hard observational constraints on the detailed compositions and optical properties of circumstellar grains, we limit ourselves to considering metallic iron $\left(\mathrm{Fe}\right.$, bulk density $\left.=7.87 \mathrm{~g} \mathrm{~cm}^{-3}\right)$, amorphous olivine $\left(\mathrm{MgFeSiO}_{4}\right.$, bulk density $=3.71 \mathrm{~g}$ $\left.\mathrm{cm}^{-3}\right)$, and water ice $\left(\mathrm{H}_{2} \mathrm{O}\right.$, bulk density $\left.=1 \mathrm{~g} \mathrm{~cm}^{-3}\right)$. These cosmically abundant materials span a wide range in condensation temperature (and therefore stellocentric distance), and in the cases of silicates and water ice, their existence is confirmed by spectroscopic observations (see, e.g., $\S 4.3$ of this paper). In reality, protostellar disks contain many more kinds of solid-state materials than we have incorporated. We have experimented with including additional grain compositions (e.g., graphite, organics, and troilite), but in no instance do we find our conclusions

TABLE 1

INPUT PARAMETERS OF REFINED MODEL

\begin{tabular}{|c|c|c|}
\hline Symbol & Meaning & Standard Value \\
\hline$M_{*} \ldots \ldots \ldots$ & stellar mass & $0.5 M_{\odot}$ \\
\hline$R_{*}^{*} \cdots \cdots \cdots$ & stellar radius & $2.5 R_{\odot}$ \\
\hline$T_{*} \ldots \ldots \ldots \ldots$ & stellar effective temperature & $4000 \mathrm{~K}$ \\
\hline$\Sigma_{0}^{*} \ldots \ldots \ldots$ & surface density at $1 \mathrm{AU}$ & $10^{3} \mathrm{~g} \mathrm{~cm}^{-2}$ \\
\hline$p \ldots \ldots \ldots \ldots$ & $-d \log \Sigma / d \log a$ & 1.5 \\
\hline$a_{o} \ldots \ldots \ldots$ & outer disk radius & $8600 R_{*}=100 \mathrm{AU}$ \\
\hline$H / h \ldots \ldots .$. & visible photospheric height/gas scale height & 4.0 \\
\hline$q_{i} \ldots \ldots \ldots$ & $-d \log N / d \log r$ in interior & 3.5 \\
\hline$q_{s} \ldots \ldots \ldots$ & $-d \log N / d \log r$ in surface & 3.5 \\
\hline$r_{\max , i} \ldots \ldots$ & maximum grain radius in interior & $1000 \mu \mathrm{m}$ \\
\hline$r_{\max , s} \cdots \cdots$ & maximum grain radius in surface & $1 \mu \mathrm{m}$ \\
\hline$T_{\text {sub }}^{\operatorname{maxh}} \ldots \ldots \ldots$ & iron sublimation temperature & $2000 \mathrm{~K}$ \\
\hline$T_{\text {sub }}^{\text {sil }} \ldots \ldots$. & silicate sublimation temperature & $1500 \mathrm{~K}$ \\
\hline$T_{\text {sub }}^{\text {ice }} \ldots \ldots . . .$. & $\mathrm{H}_{2} \mathrm{O}$ ice sublimation temperature & $150 \mathrm{~K}$ \\
\hline$M_{\text {DISK }}^{\mathrm{a}} \cdots \cdots$ & total disk mass (gas + dust) & $0.014 M_{\odot}$ \\
\hline
\end{tabular}

a The total disk mass is not an explicitly inputted parameter but is derived from $\Sigma_{0}$, $p, a_{o}$, and the inner disk cut-off radius, $a_{i}=2 R_{*}$. 
changed qualitatively. Our goal is not to be slavishly realistic but rather to highlight chief physical effects.

Thus, where local dust temperatures (=gas temperatures) fall below $T_{\text {sub }}^{\mathrm{ice}} \approx 150 \mathrm{~K}$, the grains are taken to be spheres of amorphous olivine mantled with water ice. For simplicity, the thickness of the water ice mantle, $\Delta r$, relative to the radius of the olivine core, $r$, is held constant. Where local temperatures fall between $T_{\text {sub }}^{\text {ice }}$ and $T_{\text {sub }}^{\text {sil }} \approx 1500 \mathrm{~K}$, only the pure olivine cores are assumed to remain. In innermost disk regions where local temperatures fall between $T_{\text {sub }}^{\text {sil }}$ and $T_{\text {sub }}^{\text {iron }} \approx 2000 \mathrm{~K}$, the grains are taken to be spheres of metallic iron.

The iron or silicate cores in the disk surface (interior) possess a power-law distribution of radii $r$ between $r_{\min }$ and $r_{\max , s}\left(r_{\max , i}\right)$ :

$$
d N \propto r^{-q_{s i}} d r,
$$

where $d N$ is the number density of grains having radii between $r$ and $r+d r$. Variables subscripted with " $s$ " denote quantities evaluated in the disk surface, while those subscripted with " $i$ " denote quantities evaluated in the disk interior. Our standard value of $q_{i}=q_{s}=3.5$ places most of the geometric surface area in the smallest grains and most of the mass in the largest grains. In practice, $r_{\min }$ is fixed at $10^{-2} \mu \mathrm{m}$, while $r_{\text {max }, i}, r_{\max , s}, q_{i}$, and $q_{s}$ are free to vary. Generally $r_{\max , s}<r_{\max , i}$ since large grains tend to settle quickly out of tenuous surface layers (see $\S 3.3$ of CG97). All of the cosmically abundant iron is assumed to be locked within grains. Following Pollack et al. (1994), we take 50\% of the cosmically abundant oxygen to be locked in $\mathrm{H}_{2} \mathrm{O}$ ice. Values for all cosmic abundances are obtained from Allen (2000), except for the abundance of oxygen, which is taken from Meyer, Jura, \& Cardelli (1998). Together, these assumptions yield a fractional thickness, $\Delta r / r$, for the water ice mantle equal to 0.4 .

Optical constants for amorphous olivine are obtained from the University of Jena Database (http:// www.astro.uni-jena.de; see also Jäger et al. 1994). Longward of $500 \mu \mathrm{m}$, where optical data for silicates are not available, the complex refractive index $(n+i k)$ for glassy olivine is extrapolated such that $n(\lambda \geq 500 \mu \mathrm{m})=n(500 \mu \mathrm{m})$ and $k(\lambda \geq 500 \mu \mathrm{m})=k(500 \mu \mathrm{m})(\lambda / 500 \mu \mathrm{m})^{-1}$. Optical constants for pure crystalline $\mathrm{H}_{2} \mathrm{O}$ ice are taken from the NASA ftp site (ftp:climate.gsfc.nasa.gov/pub/wiscombe/ Refrac_Index/ICE; see also Warren 1984). Though employing the constants for a cosmic mixture of amorphous ices $\left(\mathrm{H}_{2} \mathrm{O}: \mathrm{CH}_{3} \mathrm{OH}: \mathrm{CO}: \mathrm{NH}_{3}\right.$; see Hudgins et al. 1993) would be more appropriate, we nonetheless adopt the data for pure $\mathrm{H}_{2} \mathrm{O}$ ice because the latter are available over all wavelengths of interest, from the ultraviolet to the radio, whereas the former are not. One consequence of using the constants for crystalline (213-272 K) water ice as opposed to amorphous $(\sim 100 \mathrm{~K})$ ice is that spectral features due to translational lattice modes at 45 and $62 \mu \mathrm{m}$ are slightly underestimated in width and overestimated in amplitude (see, e.g., Hudgins et al. 1993). Optical constants for metallic $\mathrm{Fe}$ are obtained from Pollack et al. (1994).

The inner cutoff radius of the disk, $a_{i}$, is fixed at $2 R_{*}$. For $\mathrm{T}$ Tauri stellar parameters, this radius coincides with the distance at which iron grains in the surface layer attain their sublimation temperature. For the hotter HAe stars, the iron condensation boundary occurs at $a \approx 14-30 R_{*}$. Inside the iron condensation radius, the disk may still be optically thick to stellar radiation even if dust is absent. Opacity

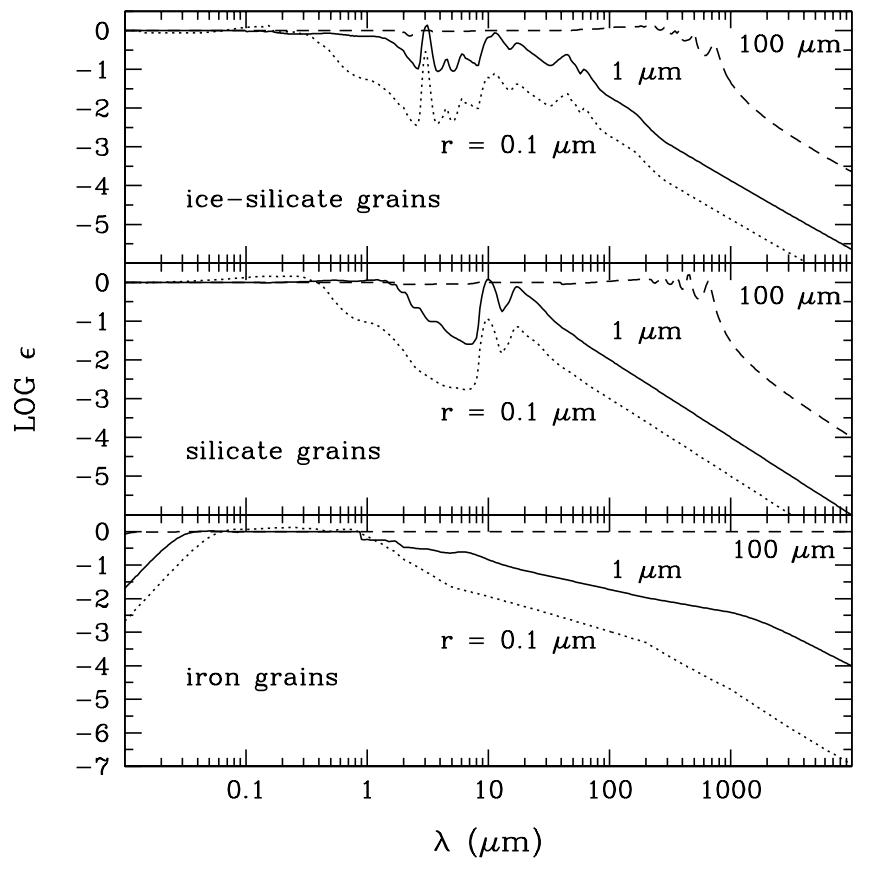

FIG. 2.-Emissivities of ice-silicate $\left(\mathrm{H}_{2} \mathrm{O} /\right.$ amorphous olivine), silicate (amorphous olivine), and iron grains having three representative core sizes. The thickness of the water ice mantle relative to the radius of the olivine core is $\Delta r / r=0.4$. Resonant features include the $\mathrm{O}-\mathrm{H}$ stretching $(3.1,4.5$ $\mu \mathrm{m})$ and $\mathrm{H}-\mathrm{O}-\mathrm{H}$ bending $(6.1 \mu \mathrm{m})$ modes in water ice; the Si-O stretching $(10 \mu \mathrm{m})$ and O-Si-O bending $(18 \mu \mathrm{m})$ modes in silicates; and the intermolecular translational $(45,62$, and $154 \mu \mathrm{m})$ modes in water ice. Oscillatory behavior near the onset of the Rayleigh limit $(2 \pi r / \lambda \approx 1)$ reflects "ripple structure " arising from our use of perfectly spherical particles.

sources include pressure-broadened molecular lines and Rayleigh scattering off hydrogen atoms (see the appendix of Bell \& Lin [1994]). For simplicity, when modeling HAe stars, we employ a one-layer blackbody disk that extends from the iron condensation boundary to $a_{i}=2 R_{*}$.

\subsection{Grain Absorption Efficiencies and Opacities}

The grain emissivity, $\varepsilon(r, \lambda)$, is equal to its absorption efficiency and is calculated using Mie-Güttler theory (Bohren \& Huffman 1983; see their subroutine BHCOAT.F). ${ }^{9}$ Figure 2 displays absorption efficiencies for our ice-silicate, silicate, and iron spheres having three representative sizes. The emissivity index in the Rayleigh limit, $\beta \equiv d \ln \varepsilon / d \ln v$, equals $1.64,2.00$, and 0.50 for the three compositions, respectively.

Well-known resonances at $\lambda \lesssim 20 \mu$ m include the $\mathrm{O}-\mathrm{H}$ stretching $(3.1,4.5 \mu \mathrm{m})$ and $\mathrm{H}-\mathrm{O}-\mathrm{H}$ bending $(6.1 \mu \mathrm{m})$ modes in water, and the Si-O stretching $(10 \mu \mathrm{m})$ and $\mathrm{O}-\mathrm{Si}-\mathrm{O}$ bending $(18 \mu \mathrm{m})$ modes in silicates.

In crystalline water ice (and crystalline silicates), optically active modes of vibration longward of $\sim 10 \mu \mathrm{m}$ are "intermolecular translational" or "intermolecular rotational." These involve collective movement of a molecule or a unit cell with respect to other molecules/unit cells in the lattice. The strengths, positions, and widths of these modes

\footnotetext{
${ }^{9}$ Our grain emissivity, $\varepsilon$, equals $Q_{\text {abs }}$ in the notation of Bohren \& Huffman (1983).
} 
are more sensitive to the presence of chemical impurities and to long range order in the solid (i.e., its degree of crystallinity, or "allotropic state") than those of fundamental stretching and bending modes at shorter wavelengths. In principle, these intermolecular modes provide information on the annealing history of initially amorphous, interstellar material in the relatively high density and high temperature environments of circumstellar disks. The intermolecular translational modes in water ice evident in Figure 2 are located at 45, 62, and $154 \mu \mathrm{m}$ (Bohren \& Huffman 1983, p. 278; Bertie, Labbé, \& Whalley 1969, Figs. 4 and 11). All of these features are positioned within the wavelength range of the Long Wavelength Spectrometer aboard ISO. Note also the blending of the $12 \mu \mathrm{m}$ intermolecular rotational band in water ice (Bohren \& Huffman 1983) with the Si-O silicate stretching mode at $10 \mu \mathrm{m}$.

Oscillatory behavior in Figure 2 near the onset of the Rayleigh limit $(2 \pi r / \lambda \approx 1)$ reflects so-called "ripple structure" that arises from our use of perfectly spherical particles (Bohren \& Huffman 1983); we expect real-world deviations from sphericity to smooth out this artificial behavior.

In the disk interior, the opacity is given by

$$
\kappa_{i}(\lambda)=\frac{\pi}{\rho_{t}} \int_{r_{\min }}^{r_{\max , i}} \frac{d N}{d r} r^{2} \varepsilon(\lambda, r) d r,
$$

where $\rho_{t}$ is the total density of gas and dust. Figure 3 displays $\kappa_{i}$ for our distribution of ice-silicate and silicate particles in cosmic abundance gas.

\subsection{Basic Equations}

In the surface layer, dust grains are directly exposed to stellar radiation. The grains attain an equilibrium tem-

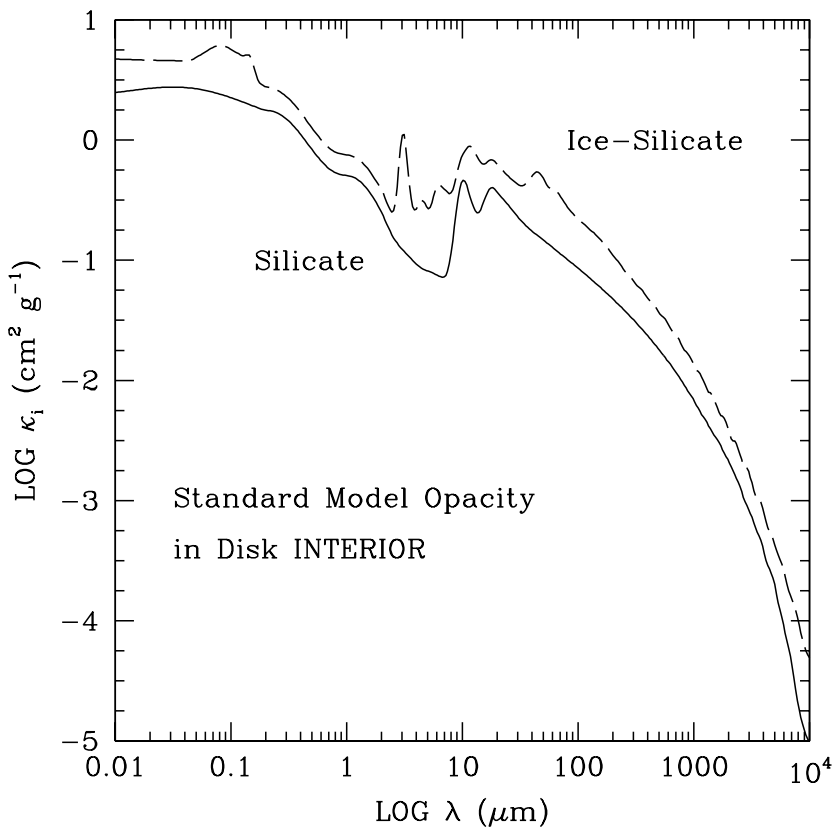

FIG. 3.-Mass absorption coefficients for our standard model distributions of ice-silicate and silicate particles in solar abundance gas. The same resonant features found in Fig. 2 are seen here. We have smoothed these curves to suppress so-called "ripple structure" at $\lambda \gtrsim 1000 \mu \mathrm{m}$ that arises from the sphericity of our particles having $r \approx 1000 \mu \mathrm{m}$. Note that these curves are sensitive to our chosen $q_{i}$ and $r_{\max , i}$. perature

$$
T_{d s}(a, r)=T_{*}\left(\frac{R_{*}}{a}\right)^{1 / 2}\left[\frac{\phi}{4} \frac{\langle\varepsilon(r)\rangle_{T_{*}}}{\langle\varepsilon(r)\rangle_{T_{d s}}}\right]^{1 / 4},
$$

where $\langle\varepsilon(r)\rangle_{T}$ is the emissivity of a grain of radius $r$ averaged over the Planck function at temperature $T$, and $\phi \approx 1 / 2$ is the fraction of the stellar hemisphere that is seen by the grain.

In our two-layer formalism, exactly half of the radiation reprocessed by the surface layer escapes directly into space. The remaining half is directed towards the disk interior. Of this latter half, a fraction, $1-e^{-\Sigma\left\langle\kappa_{i}\right\rangle}$, is absorbed by the disk interior, where $\Sigma$ is the disk surface density and $\left\langle\kappa_{i}\right\rangle_{s}$ is the opacity of the disk interior averaged over the spectrum of radiation from the surface. ${ }^{10}$ In radiative balance,

$$
\frac{\phi}{2}\left(1-e^{-\Sigma\left\langle\kappa_{i}\right\rangle_{s}}\right)\left(\frac{R_{*}}{a}\right)^{2} T_{*}^{4} \sin \alpha=\left(1-e^{-\Sigma\left\langle\kappa_{i}\right\rangle_{i}}\right) T_{i}^{4},
$$

where $T_{i}$ is the temperature of the disk interior and $\left\langle\kappa_{i}\right\rangle_{i}$ is the interior opacity averaged over the spectrum of radiation from the disk interior. ${ }^{11}$ Note that $T_{i}$ is the common temperature of interior grains of all sizes which are assumed to have thermally equilibrated with one another. Here $\alpha$ is the angle at which stellar radiation strikes the surface:

$\alpha \approx \arctan \left(\frac{d \ln H}{d \ln a} \frac{H}{a}\right)-\arctan \frac{H}{a}+\arcsin \left(\frac{4}{3 \pi} \frac{R_{*}}{a}\right)$

(cf. eq. [5] of CG97). The height of the disk photosphere, $H$, is assumed to be proportional to the vertical gas scale height, $h$, with a fixed constant of proportionality equal to 4 for our standard model. In reality, when dust and gas are well-mixed in interstellar proportions, the ratio $H / h$ decreases slowly from $\sim 5$ at 1 AU to $\sim 4$ at 100 AU. In modeling observed SEDs, we will allow $H / h$ to be a fitted constant parameter. In hydrostatic equilibrium,

$$
\frac{H}{a}=\frac{H}{h} \frac{h}{a}=\frac{H}{h} \sqrt{\frac{T_{i}}{T_{c}}} \sqrt{\frac{a}{R_{*}}},
$$

where $T_{c} \equiv G M_{*} \mu_{g} / k R_{*}$ and $\mu_{g}=3 \times 10^{-24} \mathrm{~g}$.

Equations (4) and (6) are two equations for the two unknown functions, $H(a)$ and $T_{i}(a)$. Substitution of (6) into (4) yields an algebraic equation for $T_{i}$ and the slowly varying flaring index, $\gamma \equiv d \ln H / d \ln a$. We solve this equation for $T_{i}(a)$ and $\gamma(a)$ numerically on a logarithmic grid in stellocentric distance. Our procedure is described in detail in the Appendix.

The SED of the disk equals the sum of emission from the disk interior,

$$
4 \pi d^{2} \lambda F_{\lambda, i}=8 \pi^{2} \lambda \int_{a_{i}}^{a_{o}} B_{\lambda}\left(T_{i}\right)\left(1-e^{-\Sigma \kappa_{i}}\right) a d a
$$

\footnotetext{
${ }^{10}$ In practice, we perform this average over a Planck function evaluated at the temperature of the most luminous grains in the surface. For our assumed size distribution, these dominant grains typically have radii $r \approx 0.5 \mu \mathrm{m}$; surface grains having $r \approx 0.2-1 \mu \mathrm{m}$ are responsible for absorbing $\sim 50 \%$ of the incident stellar radiation.

${ }^{11}$ We perform this average over a Planck function evaluated at temperature $T_{i}$.
} 
and from the surface layers (above and below the disk midplane),

$$
4 \pi d^{2} \lambda F_{\lambda, s}=8 \pi^{2} \lambda\left(1+e^{-\Sigma \kappa_{i}}\right) \int_{a_{i}}^{a_{o}} S_{\lambda}\left(1-e^{-\tau_{s}}\right) a d a,
$$

where $d$ is the distance to the source. The source function in the surface, $S_{\lambda}$, is the Planck function averaged over the ensemble of effective grain cross-sections in the surface layer:

$$
S_{\lambda}=\frac{2 \int_{r_{\min }}^{r_{\max }} B_{\lambda}\left(T_{d s}\right)(d N / d r) r^{2} \varepsilon(\lambda, r) d r}{\int_{r_{\min }}^{r_{\max , s}}(d N / d r) r^{2} \varepsilon(\lambda, r) d r} .
$$

In equation (9), the factor of 2 is inserted so that exactly half of the incident radiation is reprocessed by the surface layer. The normal optical depth of the surface layer, $\tau_{s}$, is similarly averaged:

$$
\tau_{s}(\lambda, a)=\frac{\int_{r_{\min }}^{r_{\max , s}}(d N / d r) r^{2} \varepsilon(\lambda, r) d r}{\int_{r_{\min }}^{r_{\max , s}}(d N / d r) r^{2}\langle\varepsilon(r)\rangle_{T_{*}} d r} \sin \alpha
$$

\section{RESULTS}

\subsection{Flaring Index}

Figure 4 displays the behavior of the flaring index, $\gamma \equiv d \ln H / d \ln a$. We conceptually divide the disk into three annular regions, as was done in CG97 (see their § 2.3.2). In

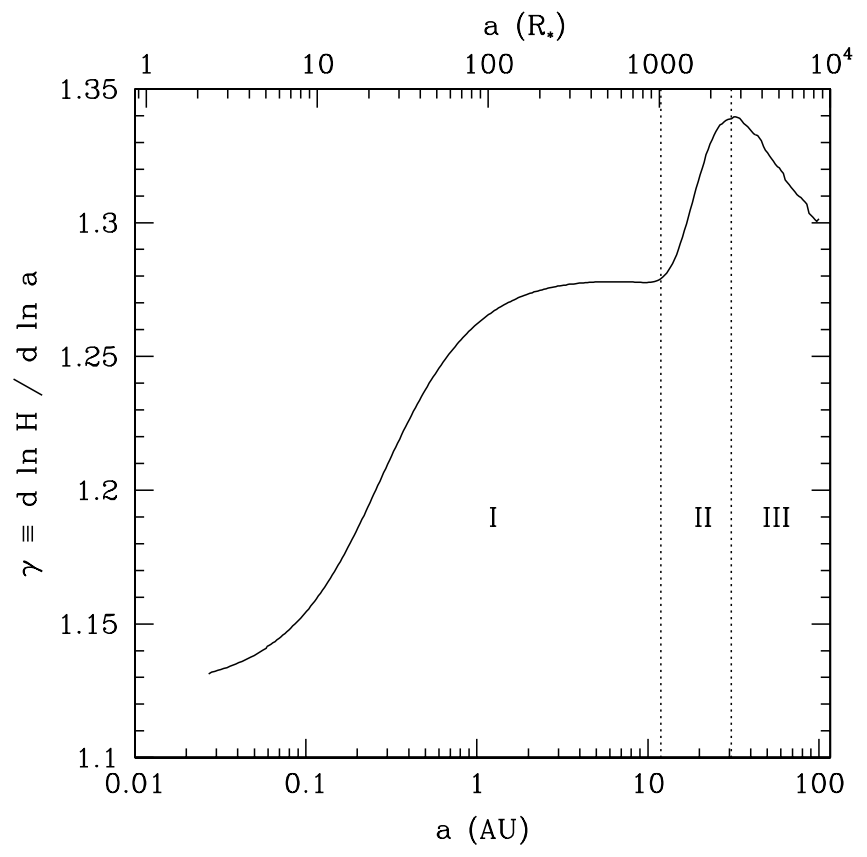

FIG. 4.-Flaring index, $\gamma \equiv d \ln H / d \ln a$, for our refined standard model. As was done in CG97 (see their $\S 2.3 .2$ ), we divide the disk into three annular regions depending on the optical depth of the disk interior. In region I, the interior behaves as a blackbody; $\gamma$ increases from its flat disk value of $1.125 \approx 9 / 8$ to its asymptotic flared value of $1.275 \approx 9 / 7$ as the disk thickness becomes increasingly larger than the stellar radius. In region II, the disk interior becomes optically thin to its own reprocessed radiation; $\gamma$ increases as interior grains enhance their temperatures to compensate for the inefficiency with which they reradiate. In region III, the interior is transparent to radiation from the surface and cools quickly with increasing distance, causing $\gamma$ to decrease. the region marked "I," the disk interior is opaque to both its own reprocessed radiation and to radiation from the surface. Here $\gamma$ increases from its flat disk value of $1.125 \approx 9 / 8$ to its asymptotic value of $1.275 \approx 9 / 7$ as the first two terms on the right-hand side of equation (5) gradually dominate the last term. In region II, the disk interior remains opaque to radiation from the surface, but is optically thin to its own reprocessed radiation. Here $\gamma$ steeply rises with $a$ because grains in the disk interior equilibrate at relatively high temperatures to compensate for the relative inefficiency with which they reradiate the incident energy. Finally, in region III, the interior is transparent to radiation from the surface (i.e., $\Sigma\left\langle\kappa_{i}\right\rangle_{s} \lesssim 1$ ); the inability of the interior to absorb the incident energy causes $\gamma$ to decrease.

\subsection{Disk Temperatures}

Figure 5 exhibits temperature profiles for the surface $\left(T_{d s}\right)$ and for the interior $\left(T_{i}\right)$. The temperatures of grains in the surface layer vary slightly with their sizes; in Figure 5, we have chosen to plot $T_{d s}$ for the size bin containing the most luminous grains, i.e., the logarithmic size interval that absorbs the greatest fraction of incident stellar radiation. For our choices of grain composition and size distribution, these dominantly absorbing grains have radii $r \approx 0.1-0.7$ $\mu \mathrm{m}$. For reference, we also overlay in Figure 5 the temperature of an imaginary blackbody sphere, $T_{\mathrm{BB}}$, which is naked before half of the stellar hemisphere.

These temperature profiles are largely similar to those found in the simpler model presented in CG97 and help to justify the approximations made there. At a given distance,

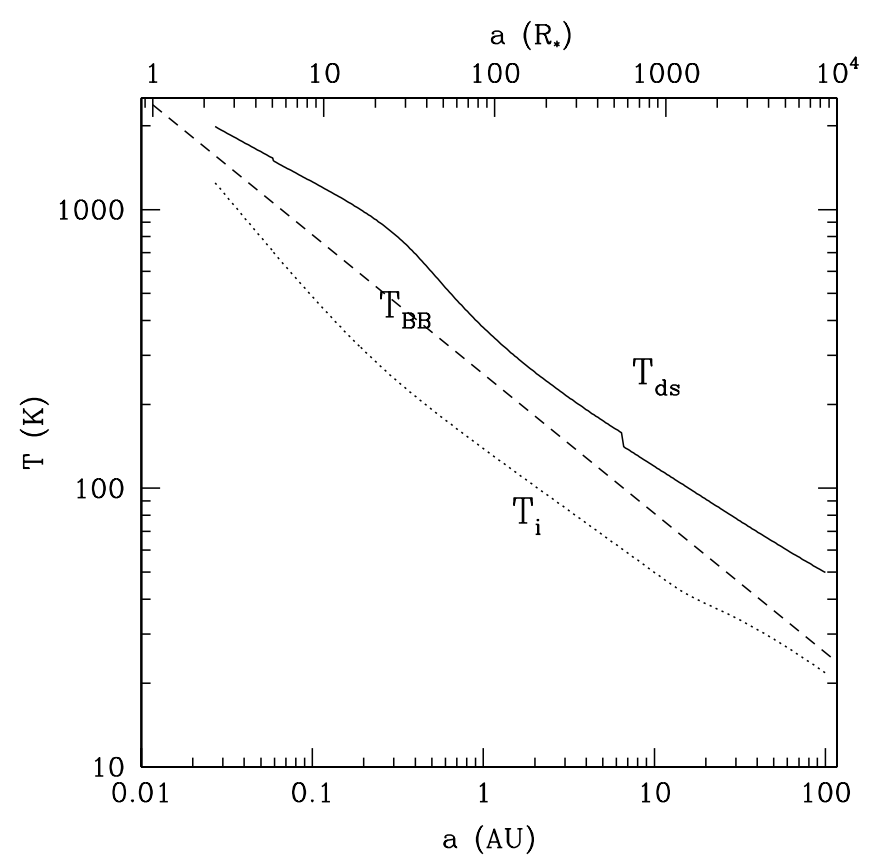

FIG. 5.-Temperature profiles for the surface and for the interior in our refined standard model. The temperatures of grains in the surface layer depend on their sizes; here, the curve marked $T_{d s}$ represents the size bin, $r \approx 0.5 \mu \mathrm{m}$, containing the most luminous grains. The discontinuity in $T_{d s}$ at $a \approx 6$ AU marks the water condensation boundary in the surface, outside of which $\mathrm{H}_{2} \mathrm{O}$ ice coats silicate cores; the discontinuity in $T_{d s}$ at $a \approx 0.05 \mathrm{AU}$ marks the silicate condensation boundary in the surface. For reference, the temperature of a spherical blackbody, which is naked before half of the stellar hemisphere, is shown as a dashed line. 
the surface is hotter than the interior by a factor of $\sim 3$. Though the interior in region "II" (see $\S 3.1$ above) is not radially isothermal as was found in the cruder analysis of CG97, $T_{i}(a)$ does flatten slightly at these distances, as expected. Deviations from a single power-law behavior for $T_{d s}(a)$ arise from structure in $\varepsilon(\lambda)$. For example, $T_{d s}$ declines slightly more steeply with $a$ for $0.5 \lesssim a_{\mathrm{AU}} \lesssim 2$ because grains at these distances cool relatively efficiently through silicate resonances at $10-20 \mu \mathrm{m}$.

\subsection{Refined Standard SED}

The SED for our refined standard model is displayed in Figure 6. The result shown here and that of CG97 (see their Fig. 6) differ primarily in the emission from the superheated surface. Here we have accounted in detail for the optical properties of a few likely circumstellar grain materials. Solid-state resonances of superheated dust grains residing in disk surface layers appear in emission. These include vibrational modes in silicates at 10 and $18 \mu \mathrm{m}$, and lattice translational modes in water ice at 45 and $62 \mu \mathrm{m}$. Emission lines from vibrational resonances in ice shortward of $10 \mu \mathrm{m}$ are absent; ice is not present in the disk surface inside 6 AU. ${ }^{12}$ Note the dearth of emission from the surface between 2 and $8 \mu \mathrm{m}$; amorphous olivine $\left(\mathrm{MgFeSiO}_{4}\right)$ grains having

\footnotetext{
${ }^{12}$ It is conceivable that vibrational resonances in water shortward of 10 $\mu \mathrm{m}$ may still appear in disk spectra if silicate particles are hydrated. Such signatures have been observed in spectra of $\mathrm{C}$ I chondritic meteorites (McSween, Sears, \& Dodd 1988).
}

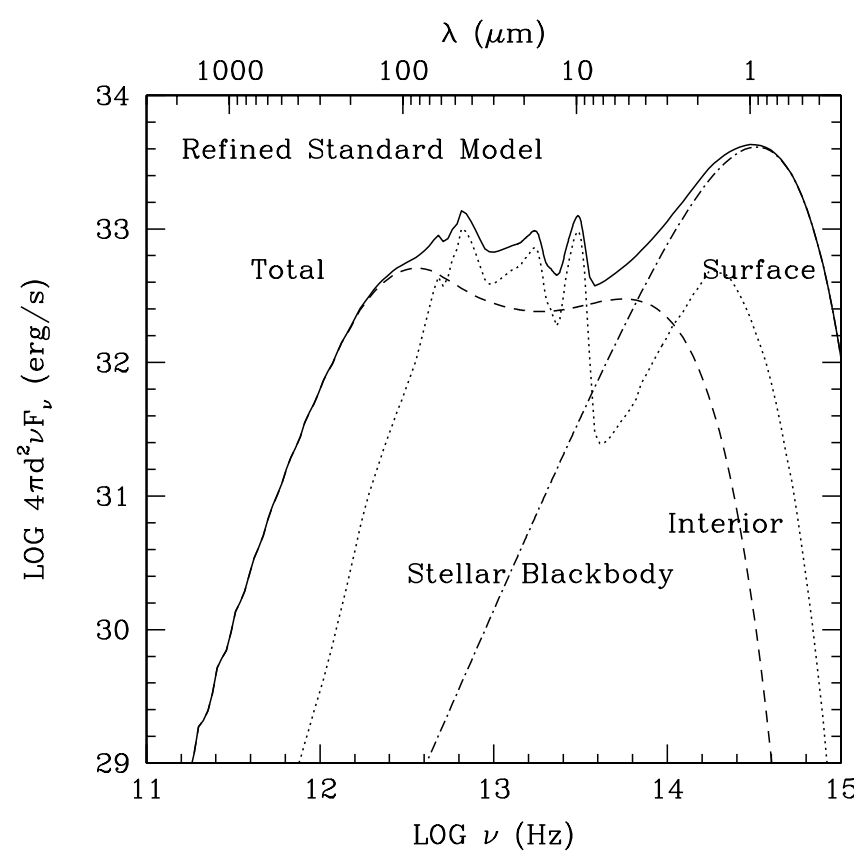

FIG. 6.-Spectral energy distribution for our refined standard model. Resonant bands of superheated dust grains in disk surface layers appear in emission. These include vibrational modes in silicates at 10 and $18 \mu \mathrm{m}$, and lattice translational modes in water ice at 45 and $62 \mu \mathrm{m}$. Absent are emission lines from vibrational resonances in ice shortward of $10 \mu \mathrm{m}$; ice is not present in the disk surface inside $6 \mathrm{AU} .{ }^{11}$ Note the dearth of emission from the surface between 2 and $8 \mu \mathrm{m}$; amorphous olivine $\left(\mathrm{MgFeSiO}_{4}\right)$ grains having $r \lesssim 1 \mu \mathrm{m}$ are relatively transparent at these wavelengths (see Fig. 2). $r \lesssim 1 \mu \mathrm{m}$ are relatively transparent at these wavelengths (see Fig. 2). The signature of this "silicate transparent region," however, is largely masked by emission from the optically thick interior at small radius. Furthermore, our computational experiments demonstrate that including other types of particles in the surface layer such as troilite (FeS) serves to further fill in this transparent region. The broad peak in disk surface emission near $\lambda \sim 1.5 \mu \mathrm{m}$ arises from our pure iron particles and iron impurities in our olivine particles.

\subsection{Dependence of SED on Input Parameters}

In Figures 7 and 8, we explore the dependence of the SED on our input parameters. In each panel, we vary the indicated parameter(s) while fixing all other parameters at their standard model values. All the variations are easily understood. We observe the following behavior:

1. Millimeter-wave fluxes are most sensitive to $\Sigma_{0}$, $p \equiv-d \ln \Sigma / d \ln a, r_{\text {max }, i}$, and $q_{i} \equiv-d \ln N / d \ln r$. The former two variables determine the amount of mass in the cool disk interior at large radius; the latter two variables affect the millimeter-wave opacity in the disk interior.

2. Millimeter-wave SEDs for $r_{\max , i}=1$ and $10 \mu \mathrm{m}$ are identical; these two cases belong to the Rayleigh limit, in which absorptive cross sections are proportional to grain volume (Bohren \& Huffman 1983). In this limit, millimeterwave opacities are independent of how the total condensable mass in water and silicates is distributed with particle size.

3. As $r_{\max , i}$ increases from 10 to $100 \mu \mathrm{m}$, the opacity at $\lambda=100-600 \mu \mathrm{m}$ in the disk interior also increases, thereby enhancing emission from the disk interior at those wavelengths. A similar effect is seen as $r_{\max , i}$ increases from 100 to $1000 \mu \mathrm{m}$.

4. For all values of $r_{\max , i}$ considered, the spectral index of the SED at $\lambda=2-4 \mathrm{~mm}$ equals $n_{2-4} \equiv d \ln \left(v F_{v}\right) / d \ln v \equiv 3$ $+\beta_{\text {eff }}=4.6$. The value of $\beta_{\text {eff }}=1.6$ equals the value of $\beta$ for our ice-silicate grains, indicating that radiation at these wavelengths emerges from optically thin material.

5. For $q_{s}=q_{i}=2.5$, most of the geometric cross section in our dust size distribution is concentrated in the largest grains $\left(r_{\max , s} \approx 1 \mu \mathrm{m}\right.$ in the surface and $r_{\max , i} \approx 1000 \mu \mathrm{m}$ in the interior). Compared to the standard model, silicate emission features from the surface at mid-infrared wavelengths are weaker because the Rayleigh limit does not apply for these large grains. At millimeter wavelengths, $n_{2-4}=4.3 \Rightarrow \beta_{\text {eff }}=1.3<\beta_{\text {ice-sil }}=1.6$; a substantial fraction of the mm-wavelength emission arises from the disk interior made optically thick by the increased number of $\mathrm{mm}$-sized particles. Note also the relative dearth of emission from $\lambda \approx 80-400 \mu \mathrm{m}$ compared to the standard model, caused by fewer numbers of $r \approx 15-70 \mu$ m sized particles in the disk interior and a concomitant loss of interior opacity at these wavelengths.

6. For $q_{s}=q_{i}=4.5$, most of the condensable mass is concentrated in the smallest grains $\left(r_{\min } \approx 0.01 \mu \mathrm{m}\right)$. Millimeter-wave opacities and therefore fluxes are lower than for our standard model. Emission at $\lambda \lesssim 100 \mu$ m does not differ from standard model results because the Rayleigh limit still applies for the most luminous grains in the surface; shapes of the emissivity curves for silicate and icesilicate grains are independent of $r$ in the Rayleigh limit (see Fig. 2). 


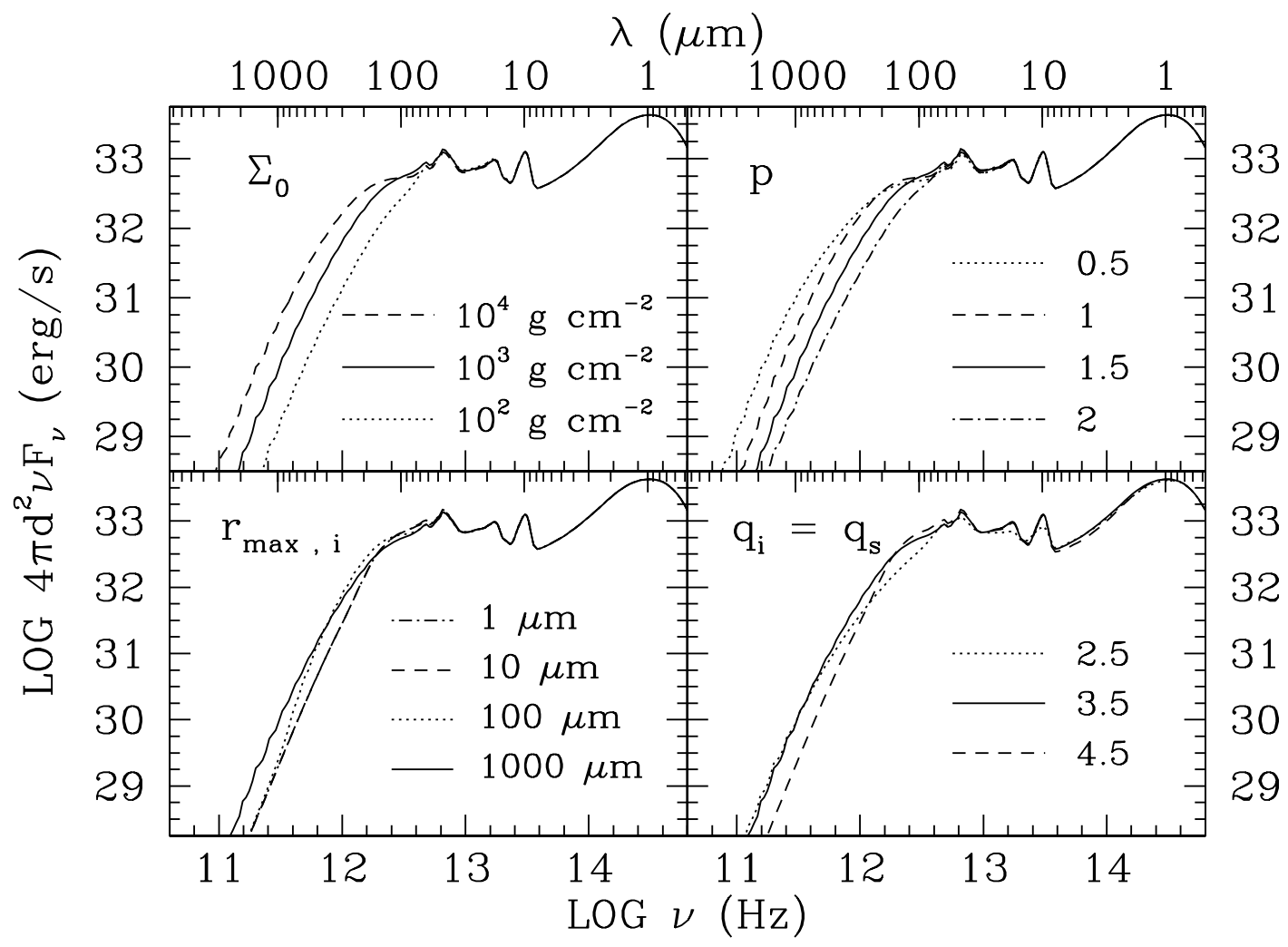

FIG. 7.-Dependence of SED on input parameters $\Sigma_{0}, p, r_{\max , i}$, and $q_{i}=q_{s}$. In each panel, the indicated parameter takes on several values, while all other parameters are fixed at their standard model values. Grain size indices $q_{i}$ and $q_{s}$ are varied simultaneously for compactness of presentation.

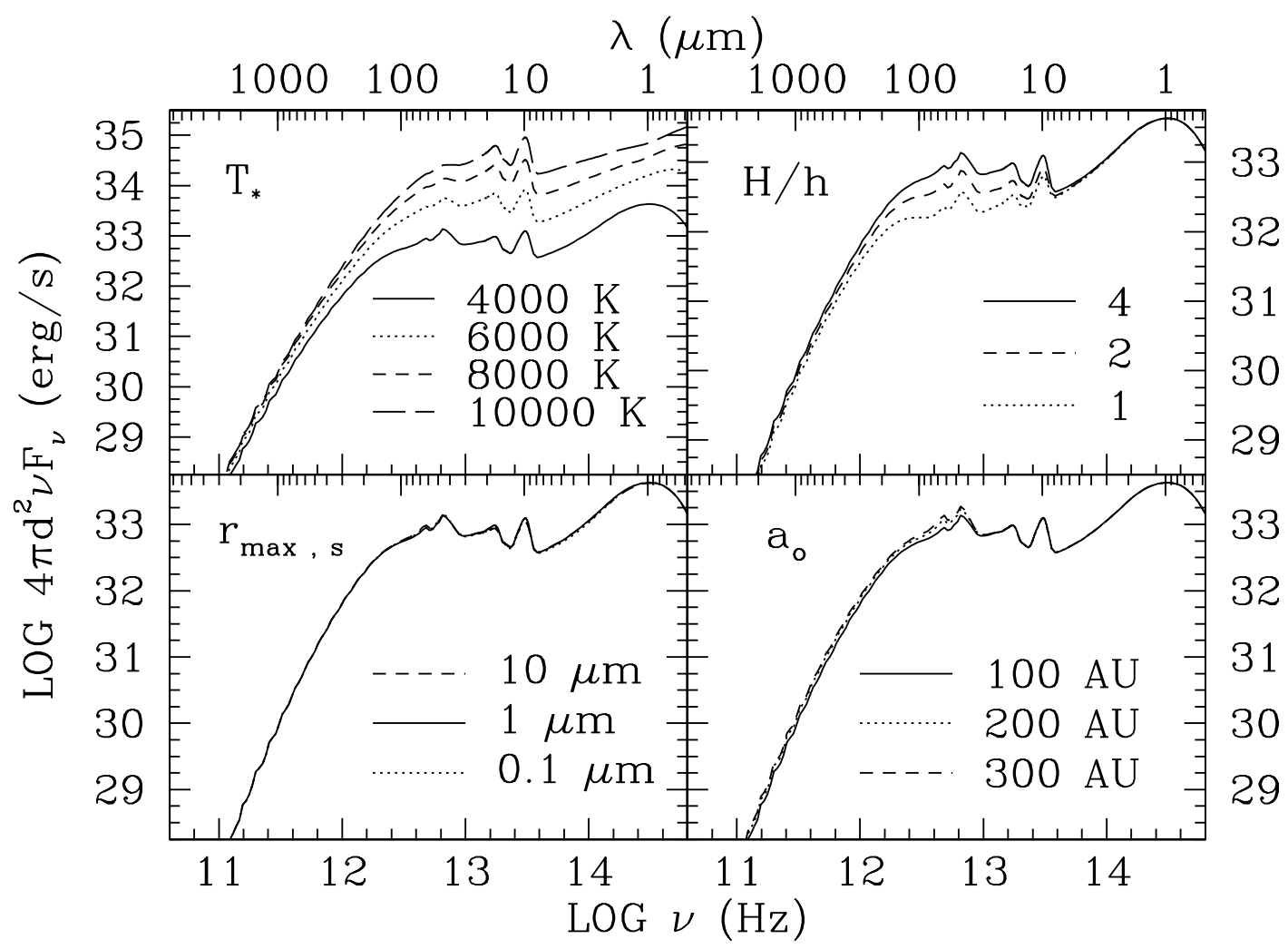

FIG. 8.-Dependence of SED on input parameters $T_{*}, H / h, r_{\max , s}$, and $a_{o}$. In each panel, the indicated parameter takes on several values, while all other parameters are fixed at their standard model values. 
7. The radial locations of condensation boundaries in disk surface layers move outward approximately as $T_{*}^{(4+\beta) / 2} \approx T_{*}^{3}$. Consequently, as $T_{*}$ increases, surface emission from water ice diminishes noticeably.

8. Reducing the height of the disk photosphere by reducing the scaling parameter $H / h$ (thereby crudely modeling the effects of vertical settling of dust) lowers the amount of stellar radiation intercepted and reprocessed by the disk. Emission at $\lambda \lesssim 200 \mu \mathrm{m}$ scales nearly linearly with $H / h$. Radiation at these wavelengths arises from the optically thick interior (region I) and from the optically thin surface; for both regimes, $v F_{v}$ scales as $\sin \alpha$ which scales approximately as $H / h$. Radiation at $\lambda \gtrsim 200 \mu \mathrm{m}$ - the RayleighJeans regime - is less sensitive to $H / h$; here the approximate scaling relation reads $v F_{v} \propto T_{i} \propto(\sin \alpha)^{0.25} \propto(H / h)^{0.25}$.

9. Surface SEDs vary negligibly with $r_{\text {max }, s}$. For our standard slope of the size distribution $\left(q_{s}=3.5\right)$, silicate and ice-silicate particles having radii $r_{*} \approx 0.5 \mu \mathrm{m}$ absorb the bulk of the radiation from the $\mathrm{T}$ Tauri star; surface grains having $r \approx 0.2-1 \mu \mathrm{m}$ are responsible for absorbing $\sim 50 \%$ of the incident stellar radiation. If $r_{\max , s}>r_{*}$, the SED is unchanged because those grains having $r \gg r_{*}$ are insufficiently numerous to be significant absorbers of radiation. If $r_{\max , s}<r_{*}$, the surface SED remains unaltered because the Rayleigh limit still obtains.

\section{FITTING SEDS OF T TAURI AND HAE STARS}

Our sample comprises three HAe and two T Tauri stars that are (1) not known to harbor stellar companions, and (2) not known to drive jets or to be surrounded by massive, $\gtrsim 500$ AU scale nebulosities that are better described by spherical envelopes rather than by flattened disks. In order of decreasing stellar effective temperature, the sample stars are MWC 480 (HD 31648), HD 36112 (MWC 758), CQ Tau (HD 36910), LkCa 15, and AA Tau. For four of our sources (MWC 480, HD 36112, CQ Tau, and AA Tau), mediumresolution $(\Delta \lambda=0.2 \mu \mathrm{m})$ ISO LWS spectra between 43 and $195 \mu \mathrm{m}$ are available. More detailed descriptions of the ISO data, including how they were reduced and what gas phase spectroscopic information they contain, are presented elsewhere (Creech-Eakman et al. 2000, in preparation). All of our sources were too weak to be observed by the ISO Short Wavelength Spectrometer.

The code that computes our refined standard model is restricted to calculating SEDs for face-on disks. A detailed study of how the SED varies with inclination, $i$, has been given previously (CG99). As described there, nonzero inclinations affect the infrared SED in two principal ways: at moderate $i$, by introducing a cosine $i$ variation in emission arising from the optically thick interior, and at extreme $i$, by blocking radiation from the disk at small radius via the intervening flared disk at large radius. The second effect on the infrared SED is negligible for our sample stars. Visual extinctions range from $A_{V}=0.3 \mathrm{mag}$ (MWC 480) to $2 \mathrm{mag}$ (CQ Tau); these modest values imply that inner disk regions are not significantly occulted by flared outer disk edges at mid-to-far infrared wavelengths. We estimate that the first effect introduces at most a factor of 2.5 overestimation in our computed fluxes between 2 and $8 \mu \mathrm{m}$ where the SED is dominated by emission from the optically thick interior. For example, the disk inclination for CQ Tau has been independently estimated to be $\sim 66^{\circ}$, based on photometric and polarimetric variability at visible wavelengths (Natta \& Whitney 2000). Such "UXOR-type" phenomena has been interpreted to arise from clumps of dust in the flared disk surface sporadically obscuring our line-of-sight to the central star.

For each source, a model SED is fitted to the ISO LWS scan (if available), millimeter wavelength fluxes, and $\sim 3-25$ $\mu \mathrm{m}$ photometric data. In three of the sources (AA Tau, CQ Tau, and MWC 480), ISO fluxes are greater than corresponding IRAS (Infrared Astronomical Satellite) fluxes at 60 and $100 \mu \mathrm{m}$ by factors of $\sim 2-3$. The origin of the discrepancies is not known. Where there are discrepancies, preference is given to the ISO LWS data for which the beam area is $\sim 2$ times smaller than that of IRAS. For HD 36112, there is excellent agreement between ISO and IRAS. Preference is given also to the central portions of the ISO scans between 50 and $170 \mu \mathrm{m}$ where individual detectors overlap in wavelength coverage and measured fluxes are consequently more reliable.

In fitting the SEDs, we fix $q_{s}=3.5$. Smaller values $\left(q_{s}<\right.$ 3) seem unlikely since they would imply that the largest grains, which tend to settle out of surface layers most quickly, dominate the geometric cross section. The SED is insensitive to larger values $\left(q_{s}>4\right)$, as shown in $\S 3.4$. We also fix $r_{\max , s}=1 \mu \mathrm{m}$ and $p=1.5$ for all models. Section 3.4 demonstrates that the SED is insensitive to $r_{\max , s}$ once $q_{s}$ is fixed at 3.5, and that $\Sigma_{0}$ and $p$ affect the SED in similar ways.

Observations and fitted theoretical models are displayed in Figures 9-13. Table 2 contains the fitted parameters for our sample. The fits are intended to be illustrative; no attempt is made to minimize fit deviations in a formal, statistical sense. The results of such an analysis would not be very meaningful anyway, since the SED tends to be degenerate with respect to simultaneous changes in several of the parameters, as we discuss in $\S 4.1$. In any case, our fitted

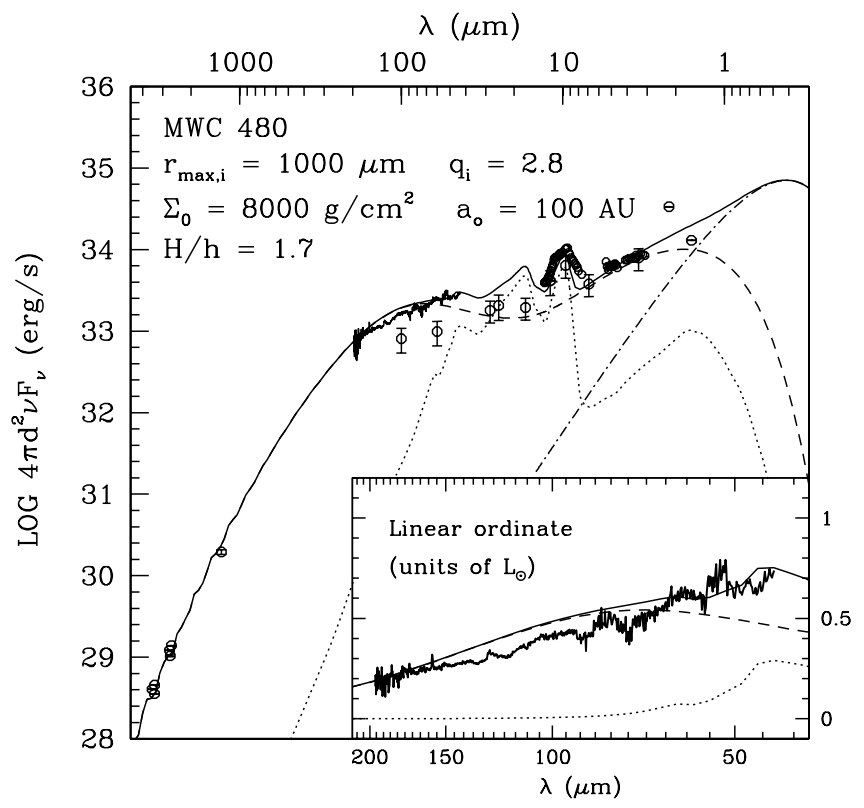

FIG. 9.- Refined two-layer model fitted to data for MWC 480. Dotted lines denote the contribution from surface layers. Dashed lines denote the contribution from the disk interior. The inset plot with linear ordinate magnifies the spectral region spanned by ISO LWS. Photometric data are taken from Mannings \& Sargent (1997), the color-corrected IRAS Point Source Catalog (1988), Sitko et al. (1999), Thi et al. (2000, in preparation), and Qi (2000). 


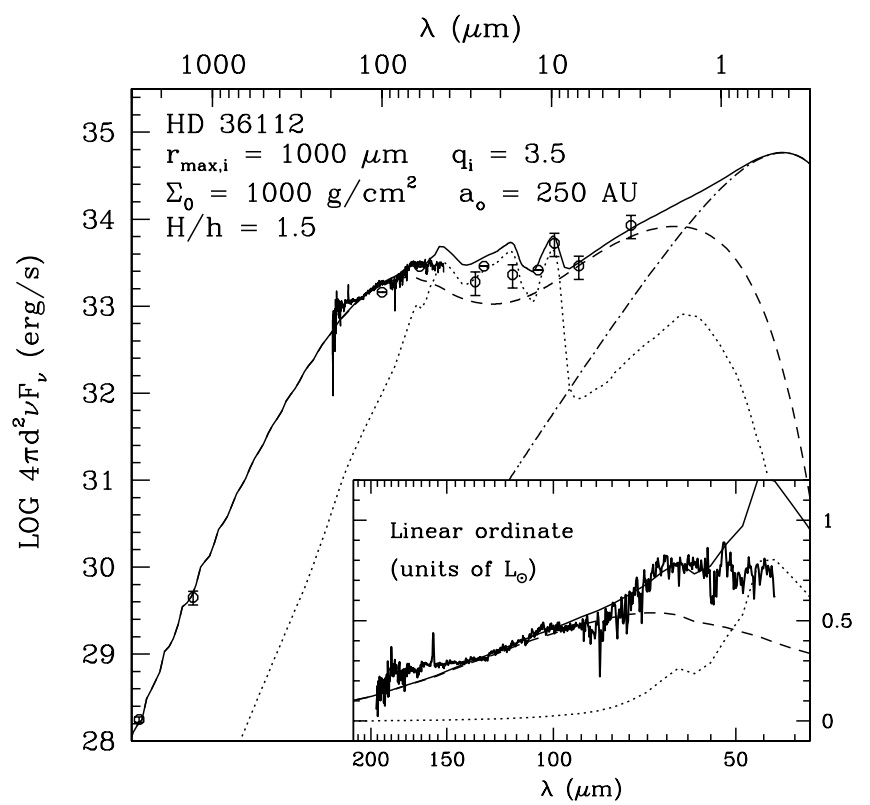

FIG. 10.- Refined two-layer model fitted to data for HD 36112. Photometric data are taken from Mannings \& Sargent (1997), the color-corrected IRAS Point Source Catalog (1988), and Thi et al. (2000, in preparation).

outer disk truncation radii for CQ Tau, HD 36112 (MWC 758), and MWC 480 are in accord with upper limits based on $\lambda=2.7 \mathrm{~mm}$ continuum images taken by Mannings \& Sargent (1997).

With few exceptions, the agreement between models and observations is good to within a factor of 2 , and serves as further evidence that simple reprocessing of central starlight by flared disks adequately explains the infrared excesses of these systems. We are aware that this is not the conclusion

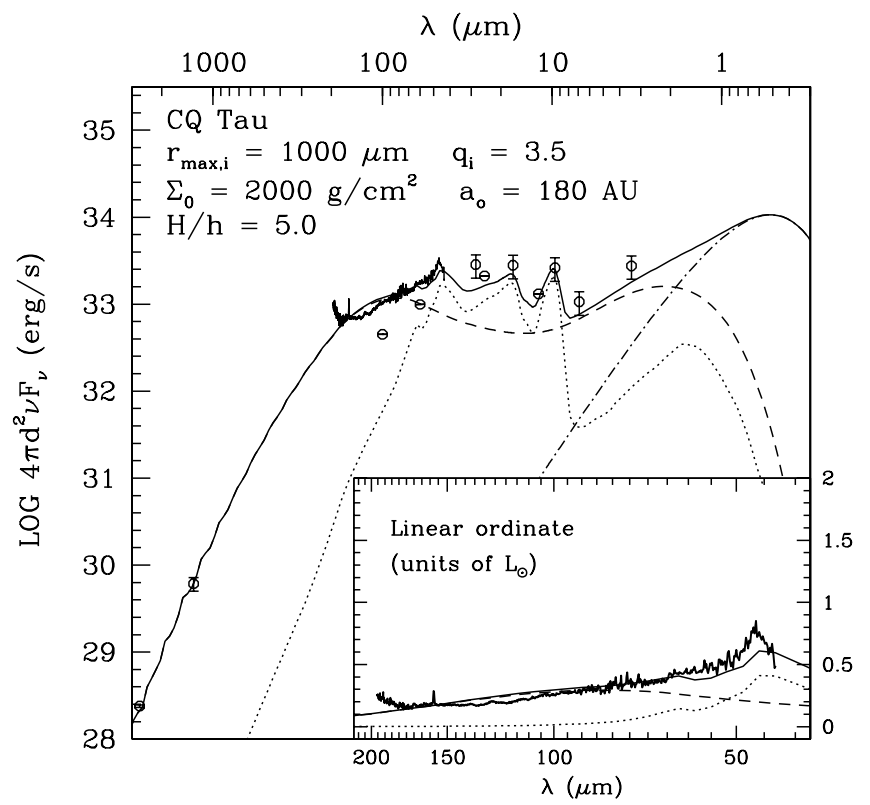

Fig. 11.- Refined two-layer model fitted to data for CQ Tau. Photometric data are taken from Mannings \& Sargent (1997), the color-corrected IRAS Point Source Catalog (1988), and Thi et al. (2000, in preparation).

of Miroshnichenko et al. (1999, hereafter MIVE99), who require the presence of tenuous envelopes having radii $\sim 1000 \mathrm{AU}$ to heat embedded HAe disks to temperatures greater than those predicted by the classical $T \propto a^{-3 / 4}$ law. This idea was first elucidated by Natta (1993) in the context of flat spectrum T Tauri stars. While some HAe systems do exhibit large-scale nebulosities whose sizes as functions of wavelength vary in accord with the calculations of MIVE99, we disagree with the statement that disks are

TABLE 2

Fitted Parameters $^{\mathrm{a}}$ of Herbig Ae and T Tauri Star/Disk Systems ${ }^{\mathrm{b}}$

\begin{tabular}{lccccc}
\hline \hline Parameter & MWC 480 & HD 36112 & CQ Tau & LkCa 15 & AA Tau \\
\hline$T_{*}(\mathrm{~K}) \ldots \ldots \ldots \ldots$ & 8890 & 8465 & 7130 & 4395 & 4000 \\
$R_{*}\left(R_{\odot}\right) \ldots \ldots \ldots \ldots$ & 2.1 & 2.1 & 1.27 & 1.64 & 2.1 \\
$M_{*}\left(M_{\odot}\right) \ldots \ldots \ldots$. & 2.3 & 2.2 & 1.7 & 1.0 & 0.67 \\
$d(\mathrm{pc}) \ldots \ldots \ldots \ldots$ & 140 & 150 & 100 & 140 & 140 \\
$\Sigma_{0}(\mathrm{~g} \mathrm{~cm}-2)^{\mathrm{c}} \ldots \ldots$. & 8000 & 1000 & 2000 & 9000 & 1500 \\
$a_{o}(\mathrm{AU})^{\mathrm{c}} \ldots \ldots \ldots \ldots$ & 100 & 250 & 180 & 200 & 250 \\
$H / h \ldots \ldots \ldots \ldots \ldots$ & 1.7 & 1.5 & 5.0 & 1.0 & 3.8 \\
$q_{i}^{\mathrm{c}} \ldots \ldots \ldots \ldots \ldots$ & 2.8 & 3.5 & 3.5 & 2.5 & 3.5 \\
$r_{\max , i}(\mu \mathrm{m})^{\mathrm{c}} \ldots \ldots \ldots$ & 1000 & 1000 & 1000 & 3000 & 1000 \\
$M_{\text {DISK }}\left(M_{\odot}\right)^{\mathrm{d}} \ldots \ldots$. & 0.11 & 0.02 & 0.04 & 0.18 & 0.03 \\
$H\left(a_{o}\right) / a_{o}{ }^{\mathrm{d}} \ldots \ldots \ldots$ & 0.13 & 0.16 & $0.45^{\mathrm{e}}$ & 0.09 & 0.58 \\
\hline
\end{tabular}

${ }^{\text {a }}$ For all sources, we fix $q_{\mathrm{s}}=3.5, r_{\mathrm{mxs}}=1 \mu \mathrm{m}$, and $p=1.5$. See text for rationale.

b Stellar parameters and distances for HAe stars are taken from Mannings \& Sargent 1997, except for CQ Tau for which $R_{*}$ and $d$ are normalized to the Hipparcos distance. Stellar parameters and distances for T Tauri stars are taken from Beckwith et al. 1990 and Webb et al. 1999.

c The continuum SED is largely degenerate with respect to simultaneous changes in $\Sigma_{0}$, $r_{\max , i}, q_{i}$, and $a_{o}$. The values shown here are not uniquely constrained.

dotal masses (in gas and dust) and maximum aspect ratios of fitted disks are derived quantities and not input parameters. As discussed in $\S 4.1$, the total disk mass depends sensitively on the a priori unknown millimeter-wave opacity and could vary by an order of magnitude.

e Our fitted maximum aspect ratio for CQ Tau and the modest visual extinction to this star ( $2 \mathrm{mag}$ ) imply a disk inclination $i \lesssim 66^{\circ}$ ( $i=0$ for face-on views). This is consistent with the independent estimation of $i \approx 66^{\circ}$ by Natta \& Whitney 2000 based on UXORtype variability at visible wavelengths. 


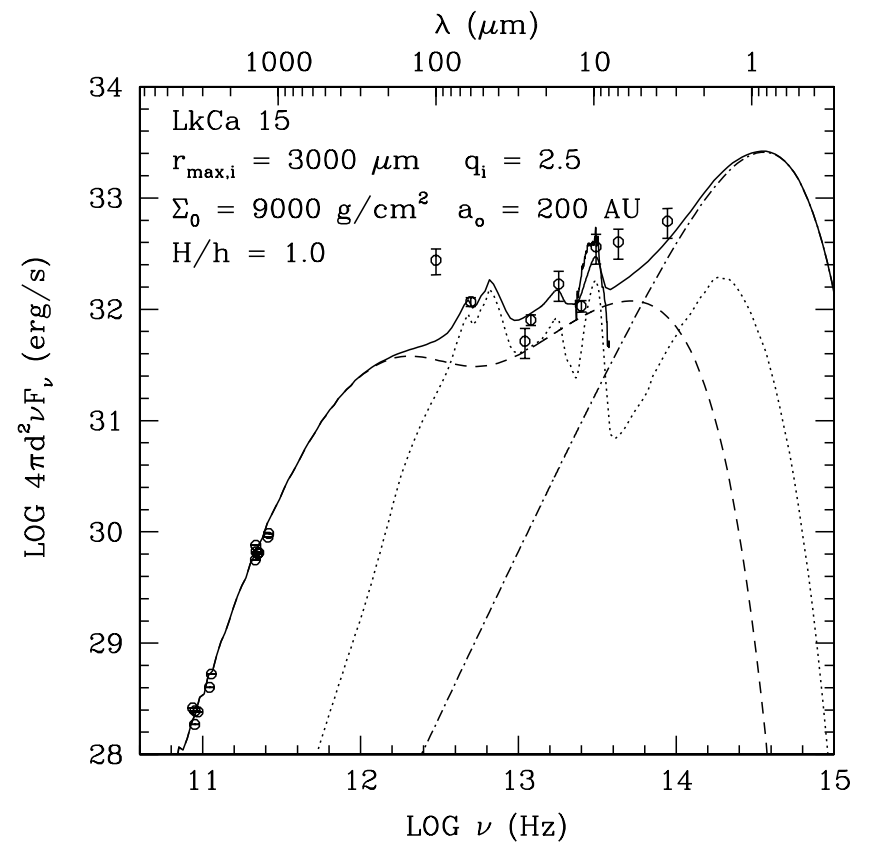

Fig. 12.- Refined two-layer model fitted to data for LkCa 15. Photometric data are taken from the color-corrected IRAS Point Source Catalog (1988), Thi et al. (2000, in preparation), and Qi (2000).

incapable of explaining the SEDs of HAe systems without heating from an envelope. Nonclassical temperature laws follow naturally from passive reprocessing of starlight by hydrostatically flared disks (e.g., Kenyon \& Hartmann 1987; CG97; this paper).

Moreover, MIVE99 claim that radiation from disk surface layers as envisioned by CG97 do not contribute substantially to SEDs of HAe stars MWC 480 and CQ Tau.

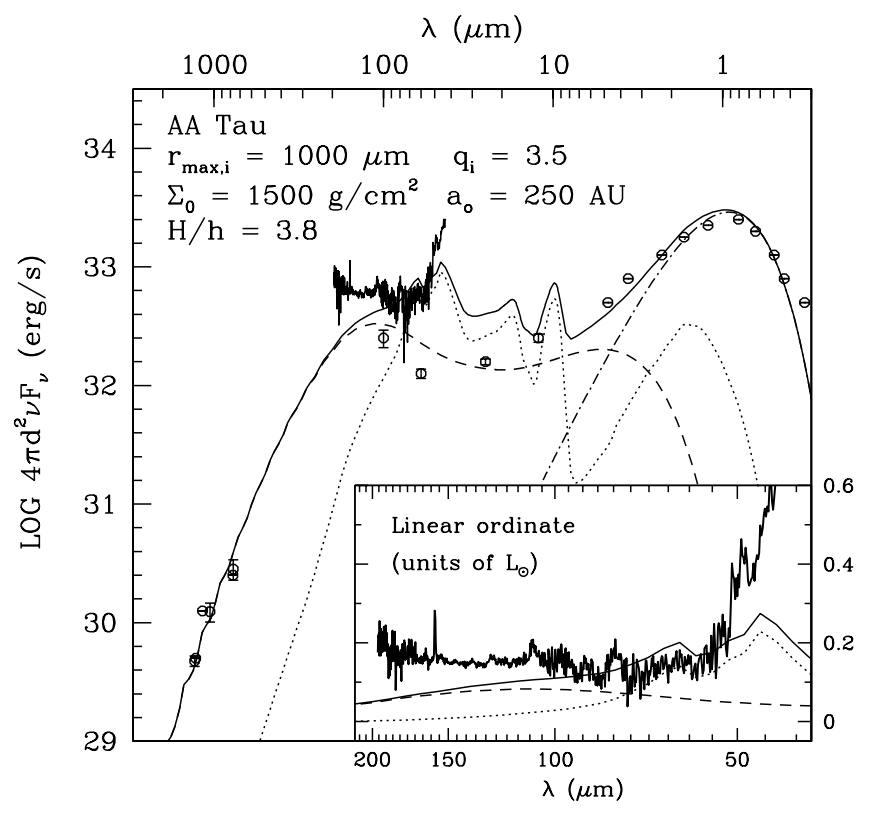

Fig. 13.-Refined two-layer model fitted to data for AA Tau. Photometric data are taken from Beckwith et al. (1990), Beckwith \& Sargent (1991), and Dutrey et al. (1996).
However, in Figures 9 and 11, our model fits to these two sources indicate that (1) emission from optically thin disk surface layers dominates emission from the optically thick disk interior between 10 and $\sim 50 \mu \mathrm{m}$, and (2) surface layer emission naturally explains the observed infrared excesses, in particular the presence of silicate and ice emission bands (see $\S 4.3$ for a more complete discussion of these bands).

Certainly the SED alone does not furnish sufficient information to uniquely constrain the geometry of dust surrounding HAe stars. We agree with MIVE99 that the presence of envelopes is an important possibility to consider for all HAe stars, and that for sources such as AB Aur their existence is persuasively implicated by imaging data at multiple wavelengths (see $\S 2$ of MIVE99). We simply wish to emphasize here that the SED alone does not rule out isolated disks heated by their central stars, and that envelopes are not the only way to achieve extra heating of the disk.

\subsection{Degeneracy between Disk Mass and Grain Size Distribution}

As might be gleaned from Figures 7 and 8, the values for $\Sigma_{0}, p, r_{\max , i}, q_{i}$, and $a_{o}$ presented in Table 2 cannot be uniquely constrained by the continuum SED alone. We display one degenerate combination in Figure 14, where two models using two different sets of parameters are fitted to the observed data for HD 36112. We feel that the fits are of comparable quality, given the crudeness of our two-layer model. It follows that the total disk mass (in dust) is highly uncertain; in fact, our two models for HD 36112 differ in their total dust mass by a factor of $\sim 6$. One relies on the

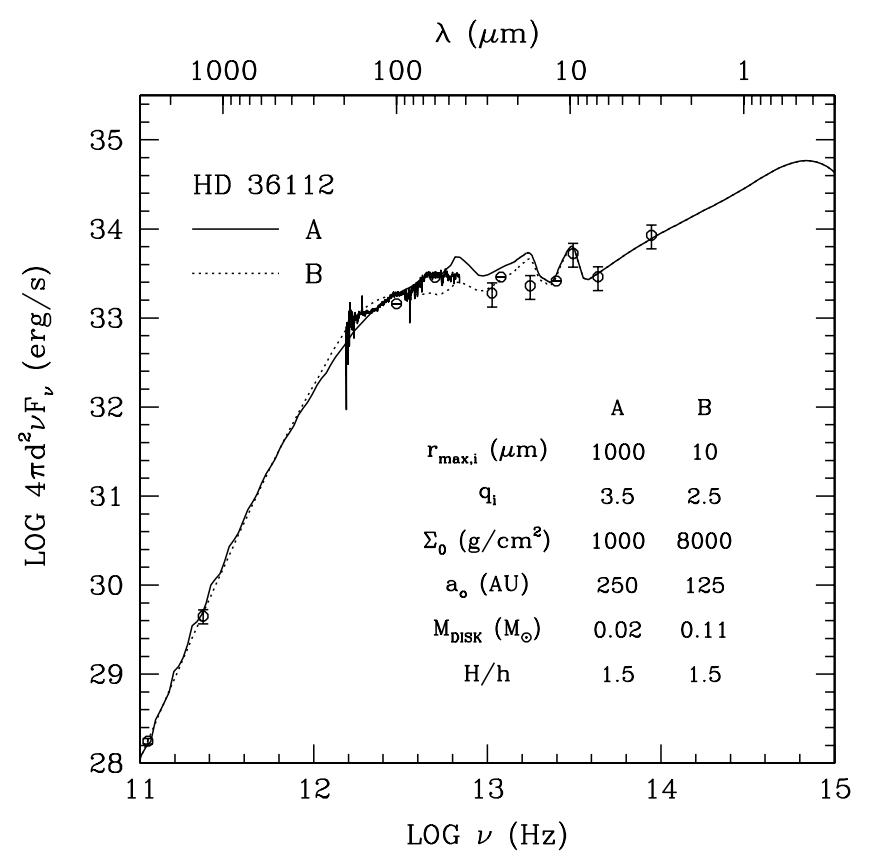

FIG. 14.-Degeneracy between surface density and grain size for HD 36112. Two models having different sets of parameters are fitted to the same data set. The disk mass, $M_{\text {DISK }}$, is not an explicitly inputted parameter but is derived from $\Sigma_{0}, p=1.5, a_{o}$, and $a_{i}=2 R_{*}$. Smaller grain sizes (i.e., smaller millimeter-wave opacities) may be traded for larger disk masses to achieve similar mm-wave optical depths and fits of comparable quality. Note, however, that both models require $H / h=1.5$ to match the overall level of infrared excess at $\lambda \lesssim 100 \mu \mathrm{m}$. 
optical thinness of the disk interior at millimeter wavelengths to measure the disk mass in dust: $M_{\text {DISK }} \propto F_{\text {mm }} \propto$ $\tau_{\mathrm{mm}} \propto \Sigma \kappa_{i}$. The problem is that the SED alone cannot disentangle $\Sigma$ from $\kappa_{i}$, which in turn depends on $q_{i}$ and $r_{\max , i}$.

The two models in Figure 14 yield significantly different results for the mass distribution in space, however. Upcoming spatially resolved observations at millimeter wavelengths will help to break this degeneracy between grain size (i.e., millimeter-wave opacity) and disk surface density (Beckwith, Henning, \& Nakagawa 2000).

\subsection{Evidence for Dust Settling}

The one disk parameter that appears to be most uniquely constrained is $H / h$, the height of the disk photosphere in units of the gas scale height. Its value is roughly proportional to the overall level of infrared excess at $\lambda \lesssim 100 \mu \mathrm{m}$. CQ Tau exhibits $H / h=5.0$, a value appropriate for gas and dust that are well-mixed in interstellar proportions. Many of our sources, however, are fitted with significantly lower values between 1 and 4 . We interpret these low values to mean that dust in disk surface layers has settled vertically towards the midplane. This was expected from the analysis of CG97 (see their § 3.3) and reinforces similar conclusions by D'Alessio et al. (1999).

The overall level of infrared excess at $\lambda \lesssim 100 \mu \mathrm{m}$ can also decrease with increasingly edge-on viewing angles, as the central portions of the disk are increasingly hidden from view by the flared outer "wall" (CG99). Inclination effects appear insufficient to explain the relatively low infrared excesses exhibited by sources here, however, because their visual extinctions are $\lesssim 1 \mathrm{mag}$ and their near-to-mid-IR fluxes are not lower than their far-IR fluxes; the reverse would be true for significantly inclined sources. Using the results from CG99, we estimate that infrared excesses may be depressed by a factor of $\sim 1.5$ for our sources owing to nonzero inclination; the suppression factor due to lower values of $H / h$ is $\sim 2-4$.

\subsection{Ice and Silicate Emission Lines}

Observational evidence for silicate emission at $10 \mu \mathrm{m}$ from the superheated surface exists for all of our sources except AA Tau. In cases where medium-resolution spectra exist (MWC 480, LkCa 15), the "trapezoidal" shape of the observed emission feature is imperfectly fitted by our model; this indicates that actual surface layer silicates have allotropic states (crystalline vs. amorphous) and compositions (pyroxene vs. olivine, and $\mathrm{Fe}: \mathrm{Mg}$ ratios) slightly different from the amorphous $\mathrm{MgFeSiO}_{4}$ that we employ. Sitko et al. (1999) reproduce the "trapezoidal" shape of the $10 \mu \mathrm{m}$ emission feature using an admixture of amorphous olivine, amorphous enstatite (a pyroxene), and crystalline olivine, in roughly equal proportions. The amplitudes of the observed emission bands indicate that the dominantly absorbing (emitting) silicates in the surface layer have sizes $r \lesssim 1 \mu \mathrm{m}$, well inside the Rayleigh limit.

Observational evidence for water ice emission at $\sim 45 \mu \mathrm{m}$ is present in ISO scans of two of the coolest stars, CQ Tau and AA Tau. The feature at $45 \mu \mathrm{m}$ represents the translational mode in water ice having the highest oscillator strength. If we examine only the ISO data in the magnified inset plots of Figures 9, 10,11, and 13, and ignore the model fits, there appears to be a trend of increasing $\sim 45 \mu \mathrm{m}$ flux relative to $\sim 55 \mu$ m continuum flux with decreasing stellar effective temperature. This behavior accords with the trend noted in $\S 3.4$ (see Fig. 8), whereby the amount of ice present in disk surface layers decreases rapidly with increasing $T_{*}$.

Notwithstanding this qualitative agreement, the model fits to water ice emission bands (or lack thereof) in the ISO spectra require substantial improvement. The best-fitted cases include (1) CQ Tau, for which there is even observational evidence of an additional translational band at 60-65 $\mu \mathrm{m}$, which our model reproduces, and (2) MWC 480, for which the high stellar temperature and the relatively small outer truncation radius of the disk suppress water ice emission to levels in approximate accord with observations. However, the observed width of the $45 \mu \mathrm{m}$ band in CQ Tau is narrower than what our model predicts. The discordancy of emission line shapes at $45 \mu \mathrm{m}$ is yet stronger in the cases of AA Tau and HD 36112. In the latter case, water ice emission is predicted by the model but is not observed. See, however, Figure 14 for an alternative model in which we substantially reduce the outer disk radius of HD 36112, thereby reducing the $45 \mu \mathrm{m}$ flux.

Accurate reproduction of observed solid-state emission features from water is hampered by a number of difficulties. Aside from the crudeness of our two-layer radiative transfer scheme, these obstacles include (1) uncertainties in the photospheric abundances of water relative to silicates (we have assumed cosmic abundances with $50 \%$ of the oxygen tied up in water and $100 \%$ of the iron locked in refractory grains); (2) uncertainties in how ice is distributed with particle size (we have assumed a constant fractional radial thickness of the ice mantle relative to the radius of the silicate core for a power-law distribution of core radii); (3) the probable presence of impurities in water ice that can shift band positions and widths; and (4) incompleteness of laboratory data for the optical constants of a cosmic mixture of ices in various allotropic states at wavelengths longward of 100 $\mu \mathrm{m}$. Improving the fits by attacking these problems is beyond the scope of our present, exploratory work.

Finally, we note the existence of several apparent emission bands in the $I S O$ spectra that we are unable to identify. Most prominent among these is a broad peak near $80 \mu \mathrm{m}$ in scans of AA Tau, MWC 480, and possibly CQ Tau and HD 36112. No resonance at $80 \mu \mathrm{m}$ exists for amorphous silicates (Jäger et al. 1994); nor is such a resonance measured for the crystalline silicates studied by Jäger et al. (1998). However, these latter authors also show that peak positions of a given vibrational mode shift towards longer wavelengths with increasing iron-to-magnesium content (higher effective vibrating masses). We propose that the $80 \mu \mathrm{m}$ feature is caused by a translational mode in crystalline olivine having an $\mathrm{Fe}: \mathrm{Mg}$ ratio intermediate between that of "natural olivine" $\left(\mathrm{Mg}_{1.96} \mathrm{Fe}_{0.04} \mathrm{SiO}_{4}\right)$ and "natural hortonolite" $\left(\mathrm{Mg}_{1.1} \mathrm{Fe}_{0.9} \mathrm{SiO}_{4}\right)$ (Jäger et al. 1998, their Table 3). If this is the case, we would expect an associated crystalline silicate translational band to appear near $51 \mu \mathrm{m}$; indeed, such an emission line does appear in ISO scans of AA Tau, MWC 480, and HD 36112. With regards to these and other perceived emission bands in the ISO data, however, the possibility of instrumental error must unfortunately be kept in mind (see $\S 4.2 .2$ of Creech-Eakman et al. 2000 , in preparation).

\subsection{Near-Infrared Excesses}

Our model near-infrared fluxes shortward of $10 \mu \mathrm{m}$ are occasionally underestimated in MWC 480, $\mathrm{LkCa} 15$, and 
AA Tau. If we adopt the disk inclination of $i \approx 66^{\circ}$ for CQ Tau (Natta \& Whitney 2000) and the resultant lowering of its model near-IR fluxes by a factor of 2.5 (see $\S 4$ ), then this star's observed near-IR fluxes are also inadequately explained. The relative dearth of surface layer emission arises partly because silicate particles are particularly transparent in this wavelength regime that exists between the vibrational resonances near $10 \mu \mathrm{m}$ and absorption due to iron impurities near $1 \mu \mathrm{m}$. It seems possible that thermally spiked emission from polycyclic aromatic hydrocarbons (PAHs) in disk surface layers may help to fill in this transparent region of the SED. The strongest resonances are due to $\mathrm{C}-\mathrm{H}$ and $\mathrm{C}-\mathrm{C}$ stretching and bending modes at 3.3, 6.2, 7.7, 8.6 and $11.3 \mu \mathrm{m}$ (Draine 1995). Upcoming high spectral resolution observations with SOFIA (Stratospheric Observatory For Infrared Astronomy) can test this hypothesis. We note, however, that Natta, Prusti, \& Krugel (1993) and Bouwman et al. (2000) argue against this explanation for HAe stars AB Aur and HD 163296 based on the need for unrealistically high abundances of PAHs and on available ISO Short Wavelength Spectrometer data.

Other neglected but possibly relevant contributions to near-IR excesses include (1) active accretion, which is likely to play an important role at disk radii inside a few AU, (2) reflected starlight off the disk surface, and (3) the possibility of dust components in addition to the disk, e.g., an optically thin, spherically distributed cloud of silicate/iron grains within a few AU of the star (MIVE99; Bouwman et al. 2000).

\section{SUMMARY}

In this work, we have constructed improved versions of two-layer passive disk models by Chiang \& Goldreich (1997). These improvements include an explicit accounting of grain size distributions and grain compositions, and numerical solution of the equations of radiative and hydrostatic equilibrium under the original two-layer approximation. We have explored how the SED varies in input parameter space and applied our models to observations of five T Tauri and Herbig Ae stars. Our principal conclusions are as follows:

1. Hydrostatically flared, passive disks having masses of $\sim 0.01-0.1 M_{\odot}$ and radii of $\sim 100-250$ AU adequately explain the infrared-to-millimeter wavelength excesses of our sample classical T Tauri and HAe stars. Unambiguous determination of the geometry of circumstellar dust requires, however, spatially resolved images. Maps from near-infrared to millimeter wavelengths generated by the Atacama Large Millimeter Array (ALMA), the Space Infrared Telescope Facility (SIRTF), and the Next Generation Space Telescope (NGST) will help to break degeneracies inherent in SEDs between, e.g., those of disks and envelopes.

2. Solid-state spectral features in the mid-infrared $(\lambda=5-60 \mu \mathrm{m})$ appear in emission from face-on disks. These emission features arise from "disk atmospheric grains": grains in disk surface layers that are directly irradiated by central starlight. The strongest resonances include the 10 $\mu \mathrm{m}$ peak from surface silicates at stellocentric distances of a few AU and the $45 \mu \mathrm{m}$ peak from surface water ice at distances of $\sim 100 \mathrm{AU}$. The strengths of these emission bands relative to that of the adjacent continuum depend on (1) the sizes of atmospheric grains that absorb the bulk of the stellar radiation, and (2) the disk viewing geometry. If atmospheric grain sizes are within the Rayleigh limit $(2 \pi r / \lambda \lesssim 1)$, emission band amplitudes saturate relative to the continuum. As atmospheric grain sizes increase beyond the Rayleigh limit $(2 \pi r / \lambda \rightarrow \infty)$, emission band amplitudes decrease. In addition, as the disk is viewed at increasingly edge-on inclinations, emission bands tend to go into absorption (CG99).

3. Values for $\Sigma_{0}, p, r_{\max , i}, q_{i}$, and $a_{o}$ influence the SED most at wavelengths longward of $100 \mu \mathrm{m}$. Their values for a given source, however, cannot be uniquely constrained by the millimeter-wave SED alone. One relies on the optical thinness of the disk interior at millimeter wavelengths to measure the disk mass in dust: $M_{\text {DIsK }} \propto F_{\mathrm{mm}} \propto \tau_{\mathrm{mm}} \propto \Sigma \kappa_{i}$. The problem is that the SED alone cannot disentangle $\Sigma$ (which depends on $\Sigma_{0}, p$, and $a_{o}$ ) from $\kappa_{i}$ (which in turn depends on $q_{i}$ and $\left.r_{\text {max }, i}\right)$. Spatially resolved millimeterwave maps can help to break the degeneracy between interior grain size and disk mass.

4. The one disk parameter that appears to be most uniquely constrained by the SED is $H / h$, the height of the disk photosphere in units of the gas scale height. Its value is roughly proportional to the overall level of infrared excess at $\lambda \lesssim 100 \mu \mathrm{m}$. CQ Tau exhibits $H / h=5.0$, a value appropriate for gas and dust that are well-mixed in interstellar proportions. Our other sources-MWC 480, HD 36112, LkCa 15, and AA Tau - are fitted with significantly lower values between 1 and 4 . We interpret these low values to mean that atmospheric grains in disk surface layers have settled vertically towards the midplane. For standard disk parameters, the time required for a 0.1 micron-sized grain to settle from $H=4 h$ to $H=0$ is $8 \times 10^{6} \mathrm{yr}$ in the absence of vertical gas flow; from $H=4 h$ to $H=h$, the required time is $8 \times 10^{5} \mathrm{yr}$ (CG97; Creech-Eakman et al. 2000, in preparation). Both these times are of the same order of magnitude as the estimated stellar ages. The actual amount of photospheric settling depends also on the unknown degree of turbulence and vertical circulation in gas.

5. Translational lattice modes in water ice appear in emission at $45 \mu \mathrm{m}$ and possibly also at $62 \mu \mathrm{m}$ in CQ Tau and AA Tau, two of the coolest stars in our sample $\left(T_{*} \lesssim\right.$ $7200 \mathrm{~K}$ ). We interpret these emission bands as arising from disk atmospheric silicates mantled by water ice at stellocentric distances of $\sim 100 \mathrm{AU}$. The hottest stars in our sample, MWC 480 and HD $36112\left(T_{*} \gtrsim 8400 \mathrm{~K}\right)$, evince no such emission bands. By itself, the dependence on stellar temperature of the location of the ice sublimation boundary in the disk surface layer is insufficiently steep (approximately $a_{\text {sub }, s} \propto T_{*}^{3}$ ) to account for the presence and absence, respectively, of water ice bands in LWS spectra of CQ Tau and HD 36112; these two stars differ in their effective temperatures by only $\sim 15 \%$.

We thank Cornelia Jäger and Ted Roush for providing optical constants of olivine and iron, respectively; Bruce Draine for suggesting that thermal spiking of PAHs might be responsible for observed near-infrared excesses; Jeroen Bouwman and Rens Waters for sending preprints of their work; Willem Schutte and Peter Goldreich for helpful discussions; and Antonella Natta for a useful referee's report. Support for E. I. C. and M. K. J. was provided by NASA grant NAG 5-7008. Additional support for E. I. C. was 
provided by NASA through a Hubble Fellowship grant awarded by the Space Telescope Science Institute, which is operated by the Association of Universities for Research in Astronomy, Inc., for NASA under contract NAS 5-26555.
M. C. E. and G. A. B. acknowledge support through the NASA Origins and ISO block grant programs. J. E. K. is supported in part by a NASA GSRP fellowship. E. F. vD. acknowledges NWO grant 614.41.003.

\section{APPENDIX A}

Solution for $T_{i}(a)$ and $\gamma(a)$

Equations (4), (5), and (6) combine to yield

$$
\begin{gathered}
\sin \left[\arctan \left(\gamma \frac{H}{h} \sqrt{\frac{T_{i}}{T_{c}}} \sqrt{\frac{a}{R_{*}}}\right)-\arctan \left(\frac{H}{h} \sqrt{\frac{T_{i}}{T_{c}}} \sqrt{\frac{a}{R_{*}}}\right)+\arcsin \left(\frac{4}{3 \pi} \frac{R_{*}}{a}\right)\right] \\
=\frac{2}{\phi}\left(\frac{1-e^{-\Sigma\left\langle\kappa_{i}\right\rangle}}{1-e^{-\Sigma\left\langle\kappa_{i}\right\rangle s}}\right)\left(\frac{T_{i}}{T_{*}}\right)^{4}\left(\frac{a}{R_{*}}\right)^{2} .
\end{gathered}
$$

This is an equation for $T_{i}(a)$, where

$$
\gamma(a) \equiv \frac{d \ln H}{d \ln a}=\frac{3}{2}+\frac{1}{2} \frac{d \ln T_{i}}{d \ln a} .
$$

Note that $\left\langle\kappa_{i}\right\rangle_{i}$ is the interior opacity averaged over the Planck function evaluated at $T_{i} ; \phi, H / h, T_{c}, T_{*}$, and $R_{*}$ are constants. We rely on the slow and modest variation of $\gamma$ with distance to solve for $T_{i}(a)$ in the following manner. We define a logarithmic grid in $a=\left\{a_{1}, \ldots, a_{N}\right\}$ where typically $N=300$. We begin by guessing a value for $\gamma$ at $a=a_{1}$. This value of $\gamma$ is used in equation (A1) to solve for both $T_{i}\left(a_{1}\right)$ and $T_{i}\left(a_{2}\right)$ by Brent's root finder (Press et al. 1992). These latter values furnish a new $\gamma \rightarrow \gamma^{\prime}=3 / 2+(1 / 2) \ln \left[T_{i}\left(a_{2}\right) / T_{i}\left(a_{1}\right)\right] / \ln \left(a_{2} / a_{1}\right)$ by (A2). This new value of $\gamma^{\prime}$ is then employed in (A1) to compute $T_{i}\left(a_{3}\right)$ and $T_{i}\left(a_{4}\right)$. These, in turn, furnish $\gamma^{\prime \prime}$ for $T_{i}\left(a_{5}\right)$ and $T_{i}\left(a_{6}\right)$. Thus the iteration proceeds by updating $\gamma$ after every two steps in distance. This procedure quickly converges to a smoothly varying solution after the first one or two iterations of $\gamma$. The initial guess of $\gamma$ at $a_{1}$ can be improved a posteriori and the calculation repeated. Updating $\gamma$ after every one step in distance introduces numerical instability. That is, if we take $\gamma^{\prime}$ to compute $\left.T_{i}\left(a_{3}\right)\right|_{\gamma^{\prime}}$, then employ $\left.T_{i}\left(a_{2}\right)\right|_{\gamma}$ and $\left.T_{i}\left(a_{3}\right)\right|_{\gamma^{\prime}}$ to calculate $\gamma^{\prime \prime}$, and so on, the resultant solution jumps erratically with every step.

\section{REFERENCES}

Allen, C. W. 2000, Allen's Astrophysical Quantities, ed. Arthur N. Cox (New York: AIP)

Beckwith, S. V. W., Henning, Th., \& Nakagawa, Y. 2000, in Protostars and Planets IV, ed. V. Mannings, A. P. Boss, \& S. S. Russell (Tucson: Univ. Arizona Press), 533

Beckwith, S. V. W., \& Sargent, A. I. 1991, ApJ, 381, 250

Beckwith, S. V. W., Sargent, A. I., Chini, R. S., \& Güsten, R. 1990, AJ, 99, 924

Bell, K. R., \& Lin, D. N. C. 1994, ApJ, 427, 987

Bertie, J. E., Labbé, H. J., \& Whalley, E. 1969, J. Chem. Phys., 50, 4501

Bohren, C. F., \& Huffman, D. R. 1983, Absorption and Scattering of Light by Small Particles (New York: Wiley)

Bouwman, J., de Koter, A., van den Ancker, M. E., \& Waters, L. B. F. M. 2000, A\&A, 360, 213

Calvet, N., Hartmann, L., \& Strom, S. E. 2000, in Protostars and Planets IV, ed. V. Mannings, A. P. Boss, \& S. S. Russell (Tucson: Univ. Arizona Press), 377-399

Calvet, N., Patiño, A., Magris, G., \& D’Alessio, P. 1991, ApJ, 380, 617

. 1992, Rev. Mexicana Astron. Astrofis., 24, 27

Chiang, E. I., \& Goldreich, P. 1997, ApJ, 490, 368 (CG97)

. 1999, ApJ, 519, 279 (CG99)

D’Alessio, P., Calvet, N., Hartmann, L., Lizano, S., \& Cantó, J. 1999, ApJ, 527,893

D’Alessio, P., Cantó, J., Calvet, N., \& Lizano, S. 1998, ApJ, 500, 411

Draine, B. T. 1995, in ASP Conf. Ser. 80, The Physics of the Interstellar Medium and Intergalactic Mediumed, ed. A. Farrara, C.F. McKee, C. Heiles, \& P. R. Shapiro (San Francisco: ASP), 133

Dutrey, A., Guilloteau, S., Duvert, G., Prato, L., Simon, M., Schuster, K., \& Ménard, F. 1996, A\&A, 309, 493

Jäger, C., Mutschke, H., Begemann, B., Dorschner, J., \& Henning, Th. 1994, A\&A, 292, 641
Jäger, C., Molster, F. J., Dorschner, J., Henning, Th., Mutschke, H., \& Waters, L. B. F. M. 1998, A\&A, 339, 904

Kenyon, S. J., \& Hartmann, L. 1987, ApJ, 323, 714

Hudgins, D. M., Sandford, S. A., Allamandola, L. J., \& Tielens, A. G. G. M. 1993, ApJS, 86, 713

Joint IRAS Science Working Group, Infrared Astronomical Satellite (IRAS) Point Source Catalog 1988 (Washington: GPO)

Lada, C. J. 1987, in IAU Symp. 115: Star-Forming Regions, ed. M. Peimbert \& J. Jugaku (Dordrecht: Reidel), 1

Lada, C. J., \& Wilking, B. A. 1984, ApJ, 287, 610

Malbet, F., \& Bertout, C. 1991, ApJ, 383, 814

Mannings, V., \& Sargent, A. I. 1997, ApJ, 490, 792

McSween, H. Y., Sears, D. W. G., \& Dodd, R. T. 1988, in Meteorites and the Early Solar System, ed. J. F. Kerridge \& M. S. Matthews (Tucson: Univ. Arizona Press), 114

Meyer, D. M., Jura, M., \& Cardelli, J. A. 1998, ApJ, 493, 222

Miroshnichenko, A., Ivezić, Z., Vinković, D., \& Elitzur, M. 1999, ApJ, 520, L115 (MIVE99)

Natta, A. 1993, ApJ, 412, 761

Natta, A., Prusti, T., \& Krugel, E. 1993, A\&A, 275, 527

Natta, A., \& Whitney, B. A. 2000, A\&A, in press

Pollack, J. B., et al. 1994, ApJ, 421, 615

Press, W. H., Teukolsky, S. A., Vetterling, W. T., \& Flannery, B. P. 1992, Numerical Recipes in Fortran (Cambridge: Cambridge Univ. Press)

Qi, C. 2000, Ph.D. thesis, California Institute of Technology

Sitko, M. L., Grady, C. A., Lynch, D. K., Russell, R. W., \& Hanner, M. S. 1999, ApJ, 510, 408

Warren, S. G. 1984, Appl. Opt., 23, 1206

Webb, R. A., et al. 1999, ApJ, 512, L63 